\title{
Restoring Tip60 HAT/HDAC2 Balance in the Neurodegenerative Brain Relieves Epigenetic Transcriptional Repression and Reinstates Cognition
}

\author{
(1) Priyalakshmi Panikker, Song-Jun Xu, * Haolin Zhang, ${ }^{\star}$ Jessica Sarthi, Mariah Beaver, ${ }^{-A v n i}$ Sheth, \\ ¿Sunya Akhter, and ๑Felice Elefant \\ Department of Biology, Drexel University, Philadelphia, Pennsylvania 19104
}

\begin{abstract}
Cognitive decline is a debilitating hallmark during preclinical stages of Alzheimer's disease (AD), yet the causes remain unclear. Because histone acetylation homeostasis is critical for mediating epigenetic gene control throughout neuronal development, we postulated that its misregulation contributes to cognitive impairment preceding $\mathrm{AD}$ pathology. Here, we show that disruption of Tip60 histone acetlytransferase (HAT)/histone deacetylase 2 (HDAC2) homeostasis occurs early in the brain of an AD-associated amyloid precursor protein (APP) Drosophila model and triggers epigenetic repression of neuroplasticity genes well before $\mathrm{A} \beta$ plaques form in male and female larvae. Repressed genes display enhanced HDAC2 binding and reduced Tip60 and histone acetylation enrichment. Increasing Tip60 in the AD-associated APP brain restores Tip60 HAT/HDAC2 balance by decreasing HDAC2 levels, reverses neuroepigenetic alterations to activate synaptic plasticity genes, and reinstates brain morphology and cognition. Such Drosophila neuroplasticity gene epigenetic signatures are conserved in male and female mouse hippocampus and their expression and Tip60 function is compromised in hippocampus from AD patients. We suggest that Tip60 HAT/HDAC2-mediated epigenetic gene disruption is a critical initial step in AD that is reversed by restoring Tip60 in the brain.
\end{abstract}

Key words: Alzheimer's disease; cognition; histone acetylation; neuroepigenetics; neuroplasticity genes; Tip60

Significance Statement

Mild cognitive impairment is a debilitating hallmark during preclinical stages of Alzheimer's disease (AD), yet its causes remain unclear. Although recent findings support elevated histone deacetylase 2 (HDAC2) as a cause for epigenetic repression of synaptic genes that contribute to cognitive deficits, whether alterations in histone acetlytransferase (HAT) levels that counterbalance HDAC2 repressor action occur and the identity of these HATs remain unknown. We demonstrate that disruption of Tip60 HAT/ HDAC2 homeostasis occurs early in the AD Drosophila brain and triggers epigenetic repression of neuroplasticity genes before $\mathrm{A} \beta$ plaques form. Increasing Tip60 in the AD brain restores Tip60 HAT/HDAC2 balance, reverses neuroepigenetic alterations to activate synaptic genes, and reinstates brain morphology and cognition. Our data suggest that disruption of the Tip60 HAT/ HDAC2 balance is a critical initial step in AD.

\section{Introduction}

Mild cognitive impairment (MCI) is a debilitating hallmark during preclinical stages of Alzheimer's disease (AD), yet the molec-

Received Sept. 28, 2017; revised March 26, 2018; accepted April 6, 2018.

Author contributions: P.P. and F.E. designed research; P.P., S.-J.X., H.Z., J.S., M.B., A.S., and S.A. performed research; P.P. and F.E. contributed unpublished reagents/analytic tools; P.P., S.-J.X., H.Z., J.S., and F.E. analyzed data; P.P. and F.E. wrote the paper.

This work was supported by the National Institutes of Health (Grant R01HD057939 to F.E.). We thank the Cell Imaging Center at Drexel University for use of their imaging facilities and Dr. Denise Garcia for generously contributing antibodies for human hippocampal immunohistochemistry.

The authors declare no competing financial interests.

*S.-J.X. and H.Z. contributed equally to this work and are co-second authors.

Correspondence should be addressed to Dr. Felice Elefant, Department of Biology, Drexel University, 3245 Chestnut Street, PISB 312, Philadelphia, PA 19104. E-mail: fe22@drexel.edu. ular causes remain unclear. Because epigenetic transcriptional control mechanisms in the brain are fundamental throughout the lifetime of a neuron and influence cognitive function (Kim et al., 2010; Meaney and Ferguson-Smith, 2010; Roth et al., 2010; Peixoto and Abel, 2013), we postulated that their misregulation contributes to the cognitive deficits that precede AD pathology (Levenson et al., 2004; Peleg et al., 2010). Histone acetylation is well characterized for its role in governing cognitive ability epigenetically by regulating synaptic gene expression via control of chromatin packaging in neurons (Borrelli et al., 2008; 
Riccio, 2010; Nelson and Monteggia, 2011). Appropriate histone acetylation homeostasis is maintained by the antagonistic activity of histone acetlytransferases (HATs) and histone deacetylases (HDACs) that activate and repress gene expression, respectively. Disruption of this fine-tuned epigenetic balance in the brain involving reduced histone acetylation levels causes transcriptional dysregulation that is a key step in AD etiology (Gräff et al., 2012; $\mathrm{Lu}$ et al., 2015). However, whether epigenetic controlled gene alterations are an initial event in $\mathrm{AD}$ that contributes to $\mathrm{MCI}$ or if they are a consequence of disease progression remains to be determined. Moreover, whereas recent findings support elevated HDAC2 as a cause for epigenetic repression of synaptic genes that contribute to cognitive deficits (Gräff et al., 2012), whether alterations in HAT levels that act to counterbalance HDAC2 repressor action occur and the identity of these HATs remain unknown.

Accumulating evidence demonstrates that impairment of cognitive ability precedes amyloid deposition by over a year in a variety of mouse AD models (Stokin et al., 2005; Steele et al., 2014), yet molecular mechanisms that trigger these deficits remain poorly understood. Synaptic defects are observable at just 4 months of age in multiple AD mouse models and, similarly, loss of synaptic connectivity is evident during early stages of the human disease (Jacobsen et al., 2006). Such preclinical AD pathologies are conserved in the $\mathrm{AD}$-associated human amyloid precursor protein (APP) Drosophila model in which deficits in synaptic plasticity in the fly neuromuscular junction (Torroja et al., 1999), axonal transport in CNS motor neurons, and apoptotic neuronal cell death in the brain are detected in third instar larvae (Sarthi and Elefant, 2011; Johnson et al., 2013) well before A $\beta$ plaques and adult lethality occur (Bolkan et al., 2012; Pirooznia et al., 2012b). Likewise, in an $\mathrm{A} \beta_{42}$ Drosophila model designed to produce $\mathrm{A} \beta_{42}$ peptide robustly in the brain to exacerbate plaque burden, onset of early memory defects precede full $\mathrm{A} \beta$ peptide aggregation by several weeks (Iijima and Iijima-Ando, 2008). We demonstrated previously that the HAT Tip60 is critical for cognitive processes based on its role in neural epigenetic gene control and, remarkably, it protects multiple cognitive neural circuits impaired in the Drosophila brain during early AD-associated neurodegenerative progression (Lorbeck et al., 2011; Pirooznia et al., 2012a; Johnson et al., 2013; Xu et al., 2014). These findings raise the possibility that disruption of Tip60 HAT function is involved in the cognition deficits that precede $\mathrm{AD}$ pathology.

Here, we tested the hypothesis that HAT Tip60 acts to counterbalance HDAC2 action in mediating epigenetic control of neuroplasticity genes and that this balance is disrupted during early stages of AD. We show that disruption of Tip60 HAT/ HDAC2 homeostasis occurs early in the AD neurodegenerative Drosophila brain and initiates epigenetic repression of neuroplasticity genes well before $\mathrm{A} \beta$ plaques and lethality occur. Repressed genes show enhanced HDAC2 binding and reduced Tip60 and histone acetylation enrichment. Remarkably, increasing Tip60 in the AD brain restores Tip60 HAT/HDAC2 balance, reverses neuroepigenetic alterations and gene repression, and reinstates brain morphology and cognition. These Drosophila neuroplasticity gene epigenetic signatures are conserved in mouse hippocampus and their expression and Tip60 HAT function are compromised in hippocampus from $\mathrm{AD}$ patients. Our findings are first to demonstrate that disruption of Tip60 HAT/HDAC2 homeostasis and concomitant epigenetically mediated neuroplasticity gene repression is a critical initial step in $\mathrm{AD}$-associated cognitive decline.

\section{Materials and Methods}

Human material. For all human studies, human hippocampal samples were purchased from MyBioSource and Amsbio, with informed consent by all donors. Control brains included one male and two females with an age range of 86-92 years. AD brains were from one male and two females with an age range of $84-90$ years. For immunohistochemical analysis, human control and AD hippocampal tissues sections were obtained and fixed in formalin and frozen. Antigen retrieval was performed on prefixed hippocampal sections. After antigen retrevial, brain slices were rehydrated and blocked with $1 \times$ blocking reagent (Roche) for $30 \mathrm{~min}$ at room temperature (RT) and incubated with primary anti-Tip60 (Abcam, ab23886), anti-NeuN (Millipore, MAB377), and anti-GFAP (Abcam, ab10062) antibody in blocking solution overnight at $4^{\circ} \mathrm{C}$. The next day, samples were washed in PBST at RT and incubated for $2 \mathrm{~h}$ at RT with Alexa-Fluor 647 and Alexa-Fluor 488. After washing three times in PBS, samples were mounted in ProLong Gold antifade reagent with DAPI (Life Technologies, P36941). Confocal microscopy was performed using an Olympus microscope with fluoview acquisition software (Olympus). Images were adjusted for size using ImageJ software.

For RT-qPCR analysis, cDNA was obtained from healthy control (RNA integrity number, RIN 8.3) and AD (RIN 8.5) brains. qRT-PCRs were performed in a $20 \mu \mathrm{l}$ reaction volume containing CDNA, $1 \mu \mathrm{M}$ Power SYBR Green PCR Master Mix (Applied Biosystems), and $10 \mu \mathrm{M}$ forward and reverse primers (for primer sequences, see extended data Fig. 2-1, available at https://doi.org/10.1523/JNEUROSCI.2840-17. 2018.f2-1). RT-qPCR was performed using an ABI 7500 Real-Time PCR system (Applied Biosystems) following the manufacturer's instructions. Fold change in messenger RNA expression was determined using the $\Delta \Delta$ Ct method.

For Western blot analysis, protein samples were electrophoresed on $10 \%$ SDS-PAGE gels and transferred to nitrocellulose membranes. Blots were blocked in TBST buffer containing 5\% nonfat milk and then incubated overnight at $4^{\circ} \mathrm{C}$ with primary anti-Tip60 (Abcam, ab23886), antiH4K5ac (Active Motif, 39699), anti-H4K12ac (Active Motif, 39166), anti-H4K16ac (Active Motif, 39168), and anti- $\beta$-actin (JLA20, DSHB, University of Iowa) antibodies diluted in TBST. Blots were washed and incubated with appropriate secondary antibodies (Odyssey) for $1 \mathrm{~h}$ at RT and scanned using a Western blot detection system (Odyssey). Total proteins were normalized to $\beta$-actin proteins. Densitometry was determined based on band intensity using ImageJ software.

Animal model. All mouse work was approved by the Institutional Animal Care and Use Committee at Drexel University. Six dissected hippocampus or tissue specificity control liver tissue samples were obtained from an equal number of mixed male and female C57BL 6-month-old control mice. Chromatin was extracted and sheared from mice hippocampus and liver using truChIP Chromatin Shearing Kit from Covaris following the manufacturer's instructions. Briefly, protein-DNA crosslinks were made at RT for 5 min with $1 \%$ formaldehyde and tissue was pulverized using the CryoPrep from Covaris. Cells were lysed and nuclei were prepared using Covaris lysis buffer. Sonication of DNA was performed using a Covaris E220 Ultrasonicator for $10 \mathrm{~min}$. The sheared chromatin was immunoprecipitated using the EZ-Magna ChIPA Chromatin Immunoprecipitation Kit (Millipore) following the manufacturer's instructions. Briefly, ChIP was performed with $10 \mu \mathrm{g}$ of sheared chromatin using Tip60 (Abcam) and H4K16ac (Active motif) antibodies. A mock reaction containing all reagents except the antibody was used as a control. Eluted material from the immunoprecipitation was purified using a QIAquick PCR purification kit (QIAGEN) and used directly for real-time PCR. Primer sets (for primer sequences, see extended data Fig. 3-1, available at https://doi.org/10.1523/JNEUROSCI.284017.2018.f3-1) were designed by NCBI/Primer-BLAST (www.ncbi.nlm. nih.gov/tools/primer-blast/). Fold enrichment of the respective genes was calculated relative to the mock no-antibody control.

Fly strains and crosses. All fly lines were raised under standard conditions at $22^{\circ} \mathrm{C}$ on standard yeasted Drosophila media (Applied Scientific Jazz Mix Drosophila Food; Thermo Fisher Scientific). The pan-neuronal driver elav ${ }^{\mathrm{C} 155}$ and the transgenic UAS lines carrying human APP 695 isoform (UAS-APP) were obtained from Bloomington Drosophila Stock 
Center. UAS-CD8::GFP;;OK107-GAL4 stock was gifted by Dr. Steven Robinow from the University of Hawaii. The generation and characterization of UAS-dTip60 ${ }^{\mathrm{E} 431 \mathrm{Q}}$ are described in Lorbeck et al. (2011). Generation and characterization of doubl- transgenic UAS APP;Tip60 ${ }^{\text {WT }}$ fly lines are described in Pirooznia et al. (2012b). The $\mathrm{w}^{1118}$ line served as the genetic background control. All experimental crosses were performed at normal physiological temperature of $25^{\circ} \mathrm{C}$ because higher temperature changes have been reported to induce nonspecific physiological and developmental alterations (Peng et al., 2007). For all experiments, transgenic expression levels for each of the UAS fly lines were assessed as described previously (Lorbeck et al., 2011; Pirooznia et al., 2012a,b; Johnson et al., 2013) to ensure that transgenic lines used for phenotype comparisons show equivalent dTip60 WT and APP expression levels.

Preparation of staged third instar larvae. Larvae were raised under standard conditions at $22^{\circ} \mathrm{C}$ on standard yeasted Drosophila media (Applied Scientific Jazz Mix Drosophila Food; Thermo Fisher Scientific). Fly embryos were collected for $2 \mathrm{~h}$ and transferred to fresh food. To ensure a homogenous population, staged larvae ( $72-76 \mathrm{~h}$ after embryo collection) were collected and used in the experiments.

Olfactory learning and memory assay. Third instar larvae were trained and tested for olfactory learning and memory performance according to protocol described in Honjo and Furukubo-Tokunaga (2005). Briefly, freshly prepared $2.5 \%$ agar plates with $2 \mathrm{ml}$ of $1 \mathrm{~m}$ sucrose (SUC) or distilled water (DW; for control) spread over the agar were used to train the third instar larvae. Then, $10 \mu$ l of undiluted odor [Linalool (LIN); Nacalai] was spotted on a filter disk and placed on the lid of the Petri dish (see Fig. 5A). Next, 50-100 larvae were transferred to the plate and kept for $30 \mathrm{~min}$. After training, the larvae were rinsed gently with DW and transferred to a test plate containing odor $(2.5 \mu \mathrm{l})$ on one side and none on the other side (see Fig. 5A). The plate was observed for $3 \mathrm{~min}$ and the responsive index (RI) was determined based on the number of larvae within the $3 \mathrm{~cm}$ semicircular area (see Fig. $5 A$ ) as follows: $\mathrm{RI}=$ (number of larvae in the odor area - number of larvae in the control area)/total number for larvae counted. $\Delta$ RI was calculated as the difference in RI of LIN/SUC and control LIN/DW. For memory performance test, the larvae were trained as mentioned above and then kept in a $2.5 \%$ agar plate for the indicated times until the olfactory test. Naive olfactory and gustatory response tests were performed as described in Honjo and Furukubo-Tokunaga (2005). To determine the speed of the larvae from different genotypes, third instar larvae were placed on $2.5 \%$ agar plates, allowed to crawl for $1 \mathrm{~min}$, and video was recorded using a Sony DCRSR47 Handycam with Carl Zeiss optics. The recorded path length was quantified and locomotion speed was calculated using Tracker software (http://physlets.org/tracker/). RIs of all the genotype were normalized using their respective locomotion speed.

$R T-q P C R$ analysis. Total RNA was isolated from 40 staged third instar larval brains using the RNeasy Plus Mini Kit (QIAGEN). Complementary DNA (cDNA) was prepared using the SuperScript II reverse transcriptase kit (Invitrogen) according to the manufacturer's instructions with $1 \mu \mathrm{g}$ of total RNA and $0.2 \mu \mathrm{g} / \mathrm{ml}$ random hexamer primers (Roche Applied Science). RT-qPCRs were performed in a $20 \mu \mathrm{l}$ reaction volume containing cDNA, $1 \mu \mathrm{M}$ Power SYBR Green PCR Master Mix (Applied Biosystems), and $10 \mu \mathrm{M}$ forward and reverse primers (for primer sequences, see extended data Fig. 2-1, available at https://doi.org/10.1523/ JNEUROSCI.2840-17.2018.f2-1). For the heterochromatin region, $4 \mu \mathrm{l}$ of the $2.5 \mu \mathrm{M}$ primer set (Active Motif, 71028) was added to a $20 \mu \mathrm{l}$ reaction volume. RT-qPCR was performed using an ABI 7500 Real-Time PCR system (Applied Biosystems) following the manufacturer's instructions. Fold change in messenger RNA expression was determined by the $\Delta \Delta$ Ct method.

ChIP and qPCR analysis. Chromatin was extracted and sheared from $\sim 100$ third instar larval heads per experiment. To obtain larval heads, the first $1 / 3$ of the larvae (anterior head region) was isolated. Remaining fat bodies were carefully dissected and discarded. All larval heads were inspected visually to ensure that the entire CNS was intact. Because we use a GAL4-inducible system to target APP expression exclusively in the nervous system of the larvae, this method ensures virtually no variability in APP expression in the samples used. For ChIPs, we used truChIP Chromatin Shearing Kit from Covaris following the manufacturer's in- structions. Briefly protein-DNA cross-links were made at RT for 5 min with $1 \%$ formaldehyde and tissue was pulverized using the CryoPrep from Covaris. Cells were lysed and nuclei were prepared using Covaris lysis buffer. Sonication of DNA was performed using a Covaris E220 Ultrasonicator for $11 \mathrm{~min}$. The sheared chromatin was immunoprecipitated using the EZ-Magna ChIP A Chromatin Immunoprecipitation Kit (Millipore) following the manufacturer's instructions. Briefly, ChIP was performed with $25 \mu \mathrm{g}$ of sheared chromatin using anti-Rpd3 (Abcam, ab1767), anti-Tip60 (Abcam, ab23886), anti-H4K5ac (Active Motif, 39699), anti-H4K12ac (Active Motif, 39166), and anti-H4K16ac (Active Motif, 39168) antibodies. A mock reaction containing all reagents except the antibody was also set up as a control. The eluted material from the immunoprecipitation was then purified using a QIAquick PCR purification kit (QIAGEN) and used directly for qPCR. Primer sets (for primer sequences, see extended data Fig. 3-1, available at https://doi.org/10. 1523/JNEUROSCI.2840-17.2018.f3-1) were designed by NCBI/PrimerBLAST (www.ncbi.nlm.nih.gov/tools/primer-blast/). Fold enrichment of the respective genes was calculated relative to the mock no-antibody control.

Western blot analysis. Protein was extracted from dissected third instar larval heads and analyzed using the BCA assay (Thermo Scientific, 23225). Protein extracts $(20 \mu \mathrm{g})$ were electrophoresed on $10 \%$ SDSPAGE gels and transferred to nitrocellulose membranes. The blots were blocked in TBST buffer containing 5\% nonfat milk and then incubated overnight at $4^{\circ} \mathrm{C}$ with primary anti-Tip60 (Abcam, ab23886), anti-Rpd3 (Abcam, ab1767), and anti- $\beta$-actin (JLA20, DSHB, University of Iowa) antibodies diluted in TBST. The blots were then washed and incubated with the appropriate secondary antibodies (Odyssey) for $1 \mathrm{~h}$ at RT and scanned using the Western blot detection system (Odyssey). The total proteins were normalized to $\beta$-actin proteins. Densitometry was determined based on band intensity using ImageJ software.

Immunohistochemistry and antibodies. Third instar larvae or adult brains were dissected in PBS, fixed in $4 \%$ paraformaldehyde in PBS, washed 3 times in PBS containing $0.1 \%$ Triton X-100 (PBST), blocked for $1 \mathrm{~h}$ at RT in phosphate-buffered Tris containing 5\% normal goat serum, and incubated with primary anti-GFP (Abcam, ab6556) or anti-elav (9F8A9, DSHB, University of Iowa) antibody in blocking solution overnight at $4^{\circ} \mathrm{C}$. Samples were washed three times in PBST at RT and incubated with Alexa-Fluor 647, Alexa-Fluor 568, or Alexa-Fluor 488 conjugated anti-HRP (Jackson Immunoresearch, 123-545-021) for $2 \mathrm{~h}$ at RT. After washing three times in PBS, samples were mounted in Vectasheild (Vector Laboratories).

Imaging and quantification. Confocal microscopy was performed using an Olympus microscope with fluoview acquisition software (Olympus). Images were displayed as projections of $1 \mu \mathrm{M}$ serial $Z$-sections. Consecutive subsets of the $Z$-stacks were used for the final projection. Images were adjusted for brightness and contrast using the ImageJ program to more clearly define mushroom bodies (MBs) and the whole brain. The area of the MB and the whole brain in the different genotypes was measured using ImageJ software.

Experimental design and statistical analysis. To determine early disruption of Tip60 HAT/HDAC2 balance using Western blot analysis, we used one-way ANOVA with Tukey's post hoc analysis, with 30 third instar Drosophila larval heads per experiment and five independent experiments $(n=150)$ for each genotype (Fig. $1 A-D)$. To determine transcriptional dysregulation using RT-qPCR analysis, we used one-way ANOVA with Tukey's post hoc analysis, with 40 third instar Drosophila larval brains per experiment and three independent experiments $(n=120)$ for each genotype (Fig. 2A,B). To compare Tip60, H4K12Ac, H4K16Ac, and $\mathrm{H} 43 \mathrm{~K} 9 \mathrm{Ac}$ enrichment in $\mathrm{w}^{1118}$ control and Tip60 ${ }^{\mathrm{E} 431 \mathrm{Q}}$, unpaired Student's $t$ test was used with 100 third instar Drosophila larval heads per experiment with three independent experiments $(n=300)$ for each genotype (Figs. 3A, 4A-C). To determine Tip60, HDAC2, H4K12Ac, H4K16Ac, and H4K5Ac enrichment using ChIP-qPCR, we used one-way ANOVA with Tukey's post hoc analysis, with 100 third instar Drosophila larval heads per experiment and three independent experiments $(n=$ 300 ) for each genotype (Figs. $3 C, D, 4 D-F$ ). To compare Tip60 and H4K16Ac enrichment in mice hippocampus and liver, we used unpaired Student's $t$ test $(n=6$; Fig. $4 G, H)$. 

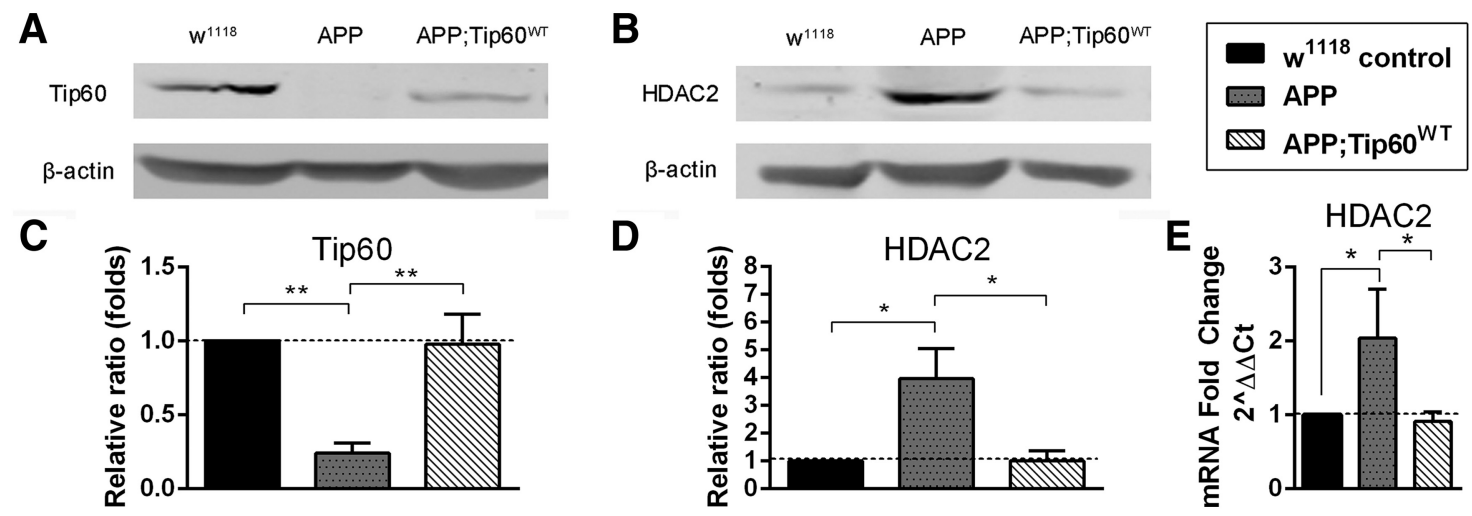

Figure 1. Disruption of Tip60 HAT/HDAC2 balance restored by increasing Tip60 HAT levels. The indicated transgene was expressed under elav ${ }^{C 155}$ pan-neuronal Gal 4 driver. $A, B$, Representative immunoblot showing Tip60 $(\boldsymbol{A})$ and HDAC2 protein levels $(\boldsymbol{B})$ from 30 staged third instar larval heads expressing indicated genotype. $\boldsymbol{C}, \boldsymbol{D}$, Densitometer quantification of $\boldsymbol{A}$ and $\boldsymbol{B}$. Data are from five independent experiments. $\boldsymbol{E}, \mathrm{RT}-\mathrm{qPCR}$ using 40 staged third instar larval brain tissue from larvae expressing the indicated genotypes under the elav ${ }^{\mathrm{C} 155}$ pan-neuronal Gal4 driver. Histogram represents relative fold change in mRNA expression level of HDAC2 relative to $\mathrm{w}^{1118}$ control flies. Data are from three independent experiments per genotype. Fold change was calculated using the $\Delta \Delta \mathrm{Ct}$ method with RP49 as a control. Statistical significance for all experiments was calculated using one-way ANOVA with Tukey's posthoc analysis. ${ }^{*} p<0.05,{ }^{* *} p<0.01$. Error bars indicate SEM.
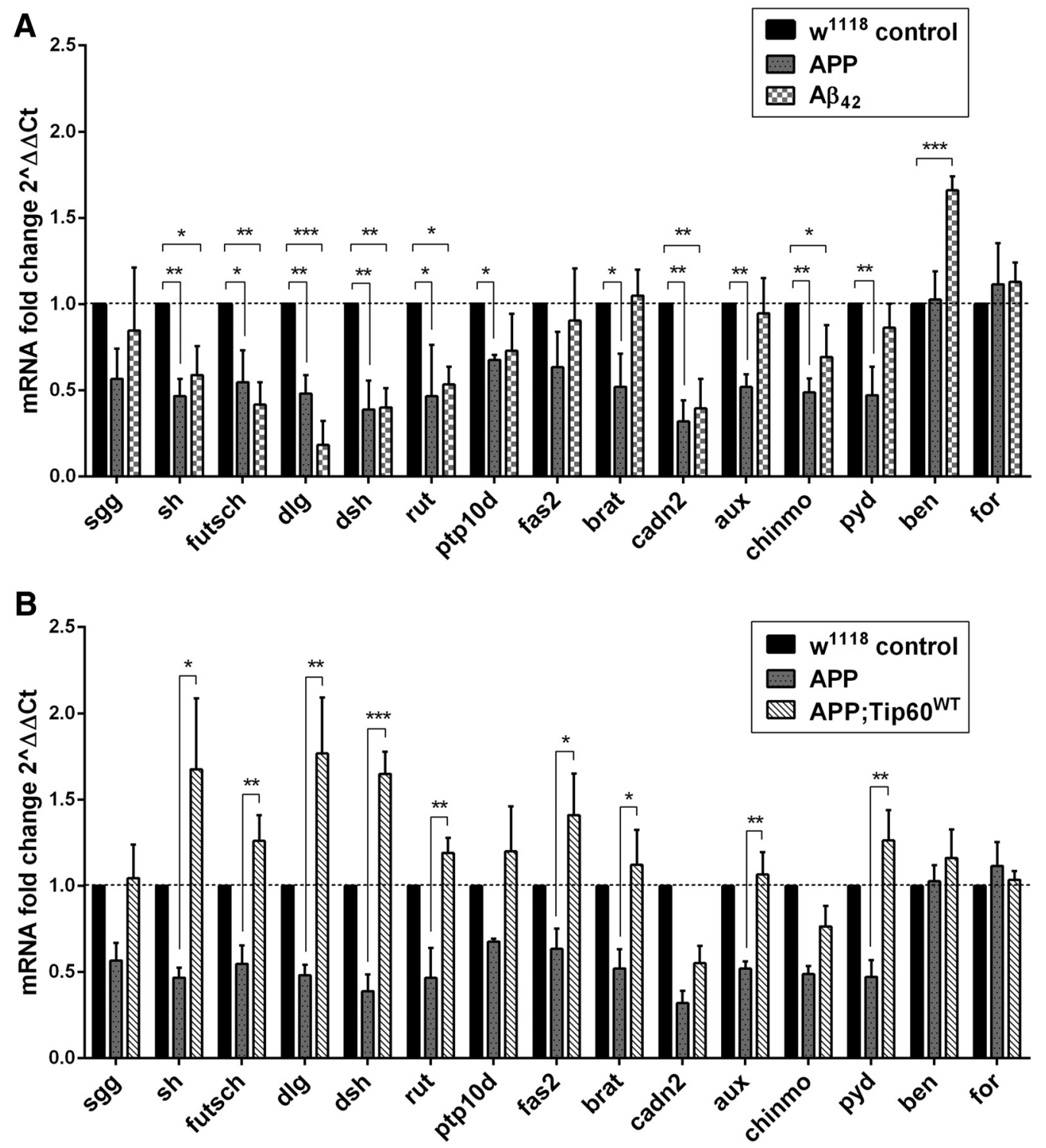

Figure 2. Repressed Tip60 neuroplasticity gene expression in the early AD brain is restored by increasing Tip60 HAT levels. RT-qPCR was performed using 40 staged third instar larval brain tissue from larvae expressing human $A P P$ and $A \beta_{42}(\boldsymbol{A})$ and human APP and APP;Tip60 ${ }^{\mathrm{WT}}(\boldsymbol{B})$ under the elav ${ }^{\mathrm{C} 155}$ pan-neuronal Gal 4 driver. Histogram represents the relative fold change in gene expression relative to $\mathrm{W}^{1118}$ control flies. Data are from three independent experiments per genotype. Fold change was calculated using the $\Delta \Delta \mathrm{Ct}$ method with RP49 as a control. Statistical significance for all experiments was calculated using one-way ANOVA with Tukey's post hoc analysis. ${ }^{*} p<0.05,{ }^{* *} p<0.01,{ }^{* * *} p<0.001$. Error bars indicate SEM. (See Figure 2-1 for primer sequences, available at https://doi.org/10.1523/JNEUROS(I.2840-17.2018.f2-1). 


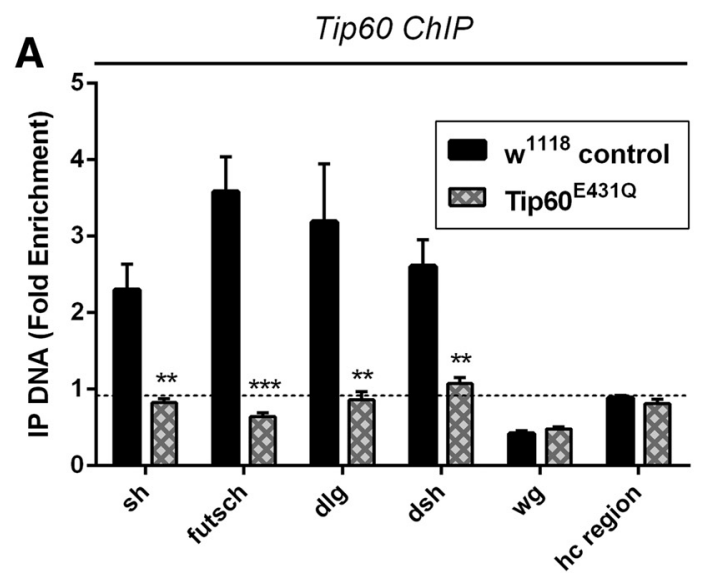

B

C

Tip60 ChIP

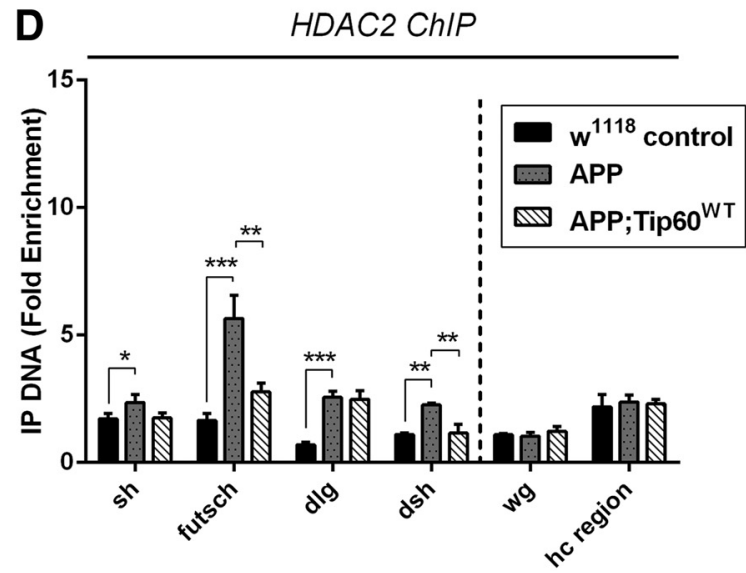

Figure 3. Tip60 reduces repressor HDAC2 recruitment and restores Tip60 binding at synaptic plasticity genes in the APP larval brain. Chromatin was isolated from 100 pooled larval heads from $\mathrm{w}^{1118}$ or Tip60 ${ }^{\mathrm{E} 4310}$ under the elav ${ }^{\mathrm{C} 155}$ pan-neuronal Gal4 driver. $\boldsymbol{A}$, Histogram representing ChIP enrichment using Tip60 antibodies. All data are from three independent experiments per genotype. Dotted line represents baseline enrichment level set by using negative control primers that amplify a fragment within a heterochromatin (hc) region within Drosophila chromosome $3 \mathrm{~L}$. Statistical significance was calculated using unpaired Student's t test. B, List of Tip60 neuroplasticity genes and their mouse and human homologs. Chromatin was isolated from 100 pooled larval heads for each indicated genotype. $\boldsymbol{C}, \boldsymbol{D}$, Histograms represents ChIP enrichment using antibodies to Tip60 ( $)$ and HDAC2 (D). All data are from three independent experiments per genotype. Dotted line separates test genes from controls. Statistical significance was calculated using one-way ANOVA with Tukey's post hoc analysis. ${ }^{*} p<0.05,{ }^{* *} p<0.01,{ }^{* * *} p<0.001,{ }^{* * * *} p<0.0001$. Error bars indicate SEM. (See Figure 3-1 for primer sequences, available at https://doi.org/10.1523/JNEUROSCI.2840-17.2018.f3-1).

For all behavioral studies, 200 Drosophila larvae were used for each genotype. To determine defects in learning ability, we used unpaired Student's $t$ test to compare LIN/SUC and LIN/DW (Fig. 5B). To determine memory retention capacity, we used two-way repeated-measures (RM) ANOVA with Sidak's post hoc analysis to compare $\mathrm{w}^{1118}, \mathrm{APP}$, and APP;Tip60 ${ }^{\text {WT }}$ larvae $(n=200)$ (Fig. $\left.5 C-F\right)$.

For quantifying larval $(n=18)$ and adult $(n=12)$ MBs (Fig. $6 A-H)$ and larval $(n=7)$ and adult $(n=12)$ whole brains (Fig. $6 I, J)$, we used one-way ANOVA with Tukey's post hoc analysis. To determine gene misregulation and Tip60-specific acetylation marks in the human $\mathrm{AD}$ hippocampus, we used unpaired Student's $t$ test with $n=3$ (Fig. 7R-T). For human samples, five hippocampal sections from three brains for each healthy control and $\mathrm{AD}$ patient were used for staining (Fig. $7 A-P$ ). For quantitation of neurons that excluded Tip60 from their nuclei, we used unpaired Student's $t$ test with $n=3$ (Fig. 7Q).

$p<0.05$ was considered statistically significant. All statistical tests were performed using GraphPad Prism version 6.00 for Windows. Degrees of freedom, $t$-values, and $p$-values are shown in the Result section and in the figures.

\section{Results}

Disruption of Tip60 HAT/HDAC2 homeostatic balance in the APP brain is an early event in AD-associated neurodegenerative progression that is restored by increasing Tip60

Disruption of a homeostatic HAT/HDAC balance within the neural epigenome involving increased HDAC2 levels and re- duced histone acetylation has been shown to cause significant cognitive impairment and is observed in multiple AD mouse models and in the hippocampus of $\mathrm{AD}$ patients (Gräff et al., 2012). However, whether there are alterations in specific HAT levels that act to counterbalance HDAC2 action and the identity of these HATs remains unknown. In addition, whether such epigenetic alterations are an early initial event in $\mathrm{AD}$ or a consequence of disease progression is unclear. To investigate whether levels of the Drosophila HDAC2 ortholog termed Rpd3 and the HAT Tip60 are altered in early stages of AD neurodegenerative progression, we measured their abundance in an $\mathrm{AD}$-associated APP Drosophila model that inducibly and neuron specifically expresses human APP. These flies show AD-related pathologies such as $\mathrm{A} \beta$ plaque accumulation in aged adult flies via the endogenous gamma (Fossgreen et al., 1998) and $\beta$-secretase cleavage pathways (Greeve et al., 2004) and, importantly, also model preclinical AD defects during the third instar larval stage that include axonal transport (Johnson et al., 2013), synaptic plasticity defects (Torroja et al., 1999), and apoptosis-driven neural cell death (Pirooznia et al., 2012b).

Using Western blot analysis on bulk proteins isolated from staged third instar larval heads expressing APP under the control of the pan neural elav-Gal4 driver, we found that, similar to studies using mouse and human AD hippocampus, APP larval heads 
A

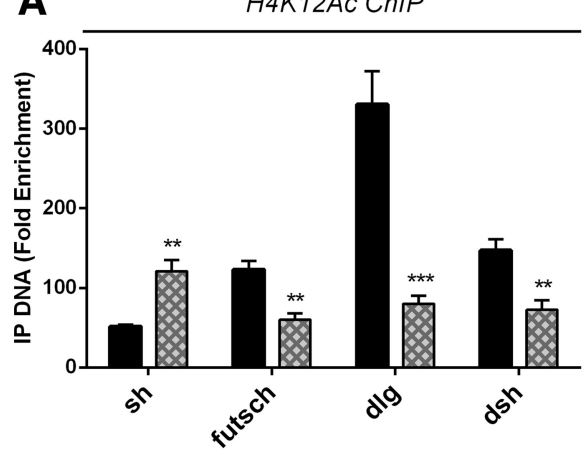

D

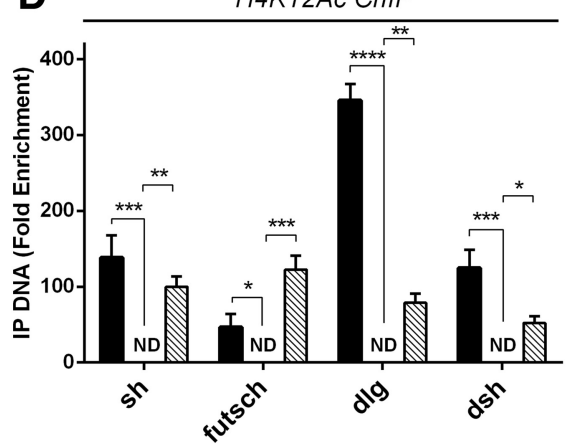

G

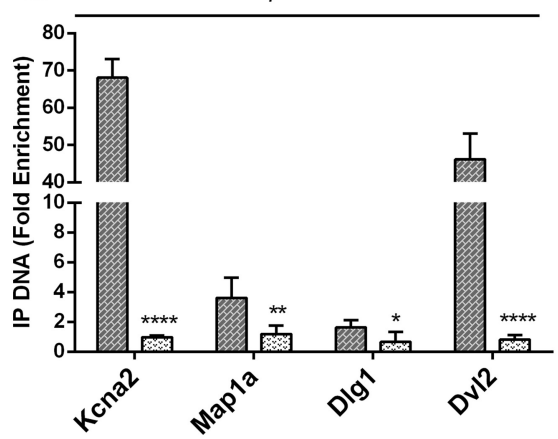

B

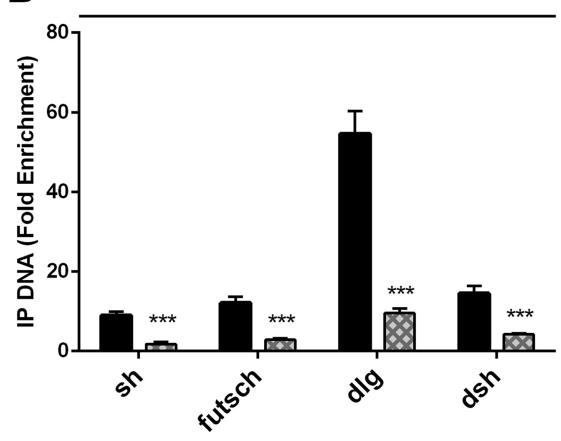

E

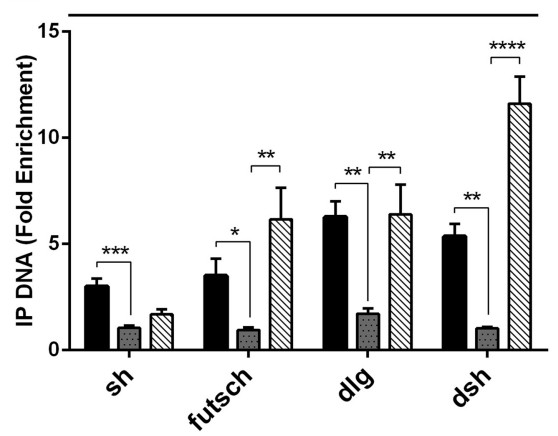

H

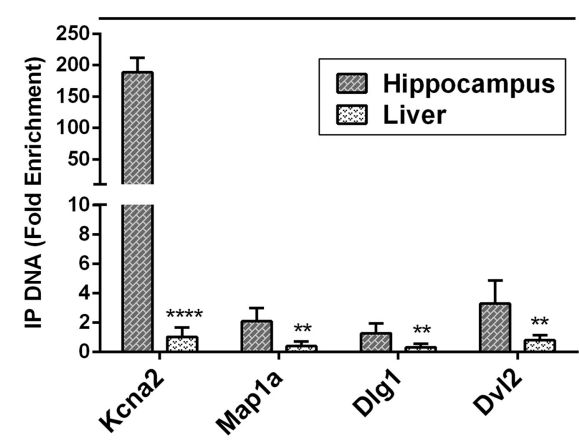

C

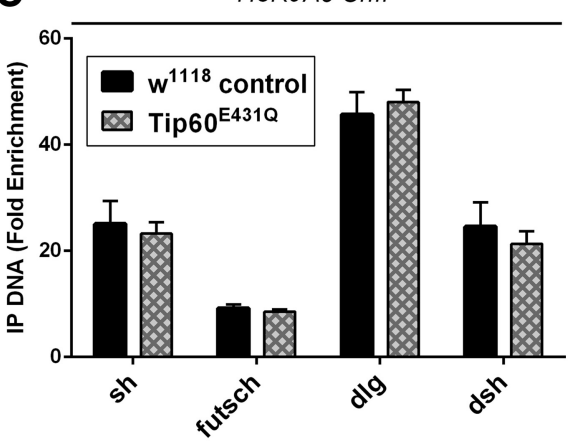

F

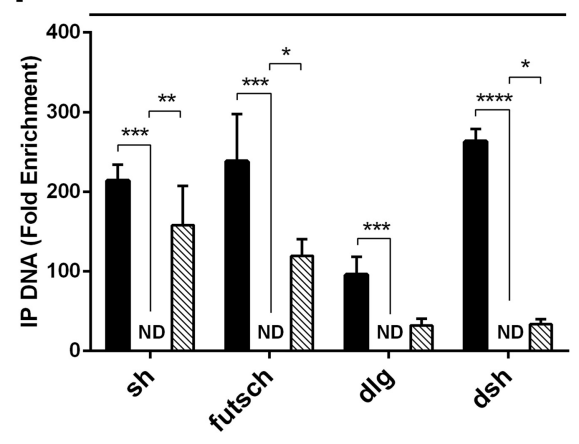

Figure 4. Tip60 restores specific epigenetic signatures at Tip60 neuroplasticity gene loci in APP larval brain. Chromatin was isolated from 100 pooled larval heads for each indicated genotype under the elav ${ }^{C 155}$ pan-neuronal Gal4 driver. $\boldsymbol{A}-\boldsymbol{C}$, Histogram representing ChIP enrichment using the H4K12Ac $(\boldsymbol{A}), \mathrm{H} 4 \mathrm{~K} 16 \mathrm{Ac}(\boldsymbol{B})$, and H3K9AC $(\boldsymbol{C})$ antibodies. All data are from three independent experiments per genotype. Statistical significance was calculated using unpaired Student's $t$ test. Chromatin was isolated from 100 pooled larval heads for each indicated genotype. $\boldsymbol{D}-\boldsymbol{F}$, Histograms representing ChIP enrichment using the H4K12Ac (D), H4K16Ac $(\boldsymbol{E})$, and H4K5Ac $(\boldsymbol{F})$ antibodies. All data are from three independent experiments per genotype. ND, Not detectable. Statistical significance was calculated using one-way ANOVA with Tukey's post hoc analysis. $\mathbf{G}, \boldsymbol{H}$, Histograms representing (hIP enrichment using Abs to Tip60 ( $\boldsymbol{G}$ ) and $\mathrm{H} 4 \mathrm{~K} 16 \mathrm{Ac}(\boldsymbol{H})$ at neuroplasticity genes in chromatin isolated from six dissected hippocampus or tissue-specific control liver tissue from equal number of mixed male and female $(57 \mathrm{BL} 6$-month-old control mice ( $n=6$ ). Statistical significance was calculated using unpaired Student's $t$ test. ${ }^{*} p<0.05,{ }^{* *} p<0.01,{ }^{* * *} p<0.001,{ }^{* * *} p<0.0001$. Error bars indicate SEM.

also displayed an increase in Rpd3 (HDAC2) protein levels (Fig. $1 B, D ; F_{(2,12)}=6.719, p=0.011$, one-way ANOVA with Tukey's post hoc analysis) compared with control wild-type $\mathrm{w}^{1118}$ flies. We also observed a reduction in Tip60 levels (Fig. $1 A, C ; F_{(2,12)}=$ $12.16, p=0.0013$, one-way ANOVA with Tukey's post hoc analysis), suggesting that, in addition to HDAC2, Tip60 HAT levels are also disrupted in the $\mathrm{AD}$ brain. We next investigated whether increasing Tip60 HAT levels in the AD larval brain could reinstate disrupted Tip60 HAT/HDAC2 balance. To test this, we assessed Tip60 and Rpd3 (HDAC2) abundance in staged third instar larval heads using our APP;Tip $60^{\text {WT }}$ fly line that expresses both APP and Tip60 ${ }^{\mathrm{WT}}$ driven by elav-Gal4 to induce a neural-specific increase of Tip60 levels in the brain during early $\mathrm{AD}$-associated neurodegenerative progression (Pirooznia et al., 2012b). As expected, Western blot analysis showed an increase in Tip60 protein levels in APP;Tip60 WT larval heads, but, surprisingly, also revealed a significant decrease in HDAC2 (Fig. $1 A-D$ ). To determine whether this decrease in Rpd3 was initiated at the level of transcription, we performed RT-qPCR analysis using APP and APP;Tip60 WT larval brains to assess Rpd3(HDAC2) mRNA levels. We observed a similar trend as in our protein analysis in that there was a significant increase in Rpd3(HDAC2) mRNA levels in the AD fly brain that were reduced upon increasing Tip60 HAT levels (Fig. $1 E ; F_{(2,6)}=7.779, p=0.0216$, one-way ANOVA with Tukey's post hoc analysis). Together, these results demonstrate that there is disruption of appropriate Tip60 HAT/Rpd3(HDAC2) homeostatic balance in the early neurodegenerative $\mathrm{AD}$ brain that is due at least in part at the level of transcriptional control for Rpd3(HDAC2) and can be alleviated by increasing Tip60 HAT levels in the brain. 
A

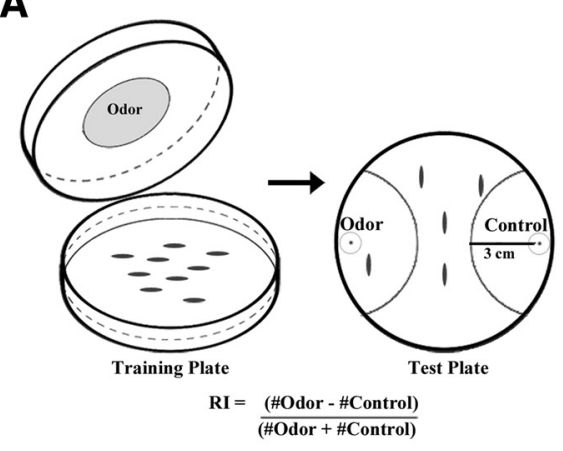

C

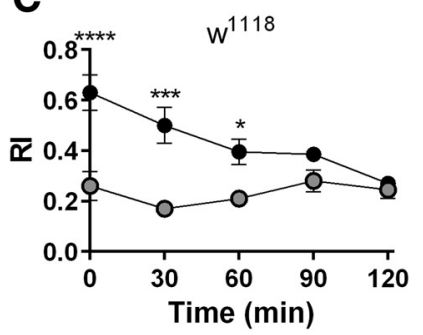

D
B
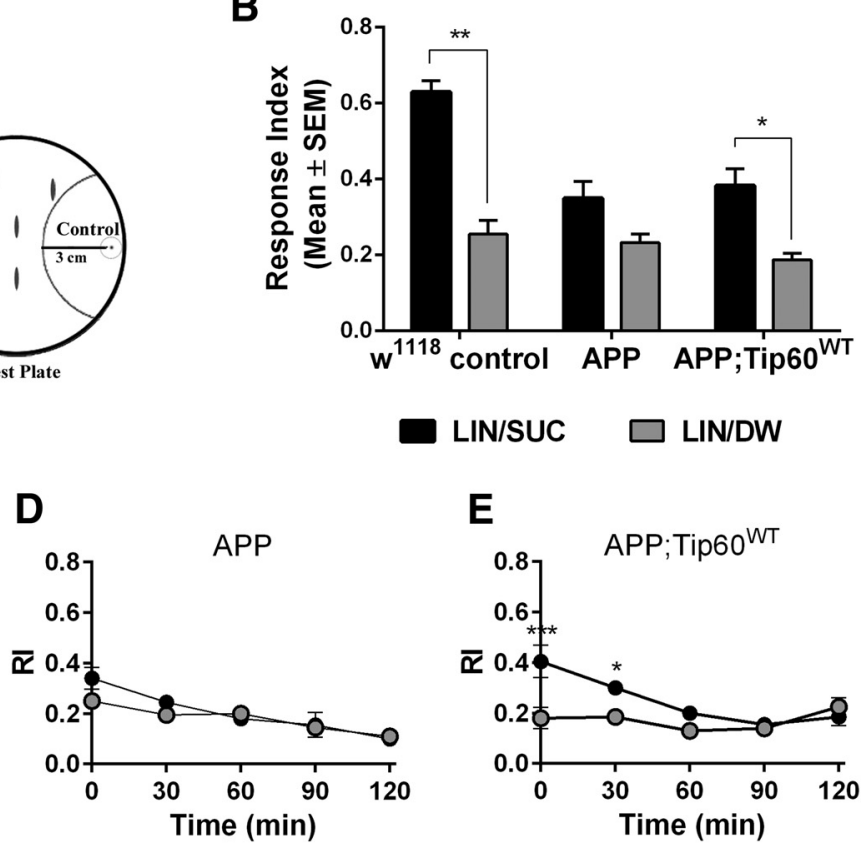

E

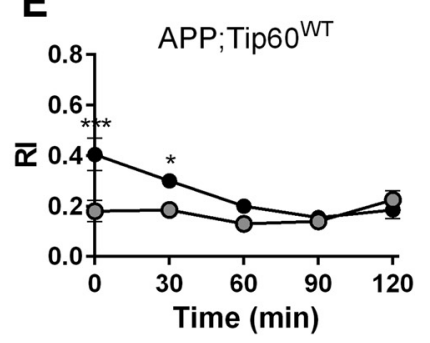

$\mathbf{F}$

LIN/SUC

- L LIN/DW

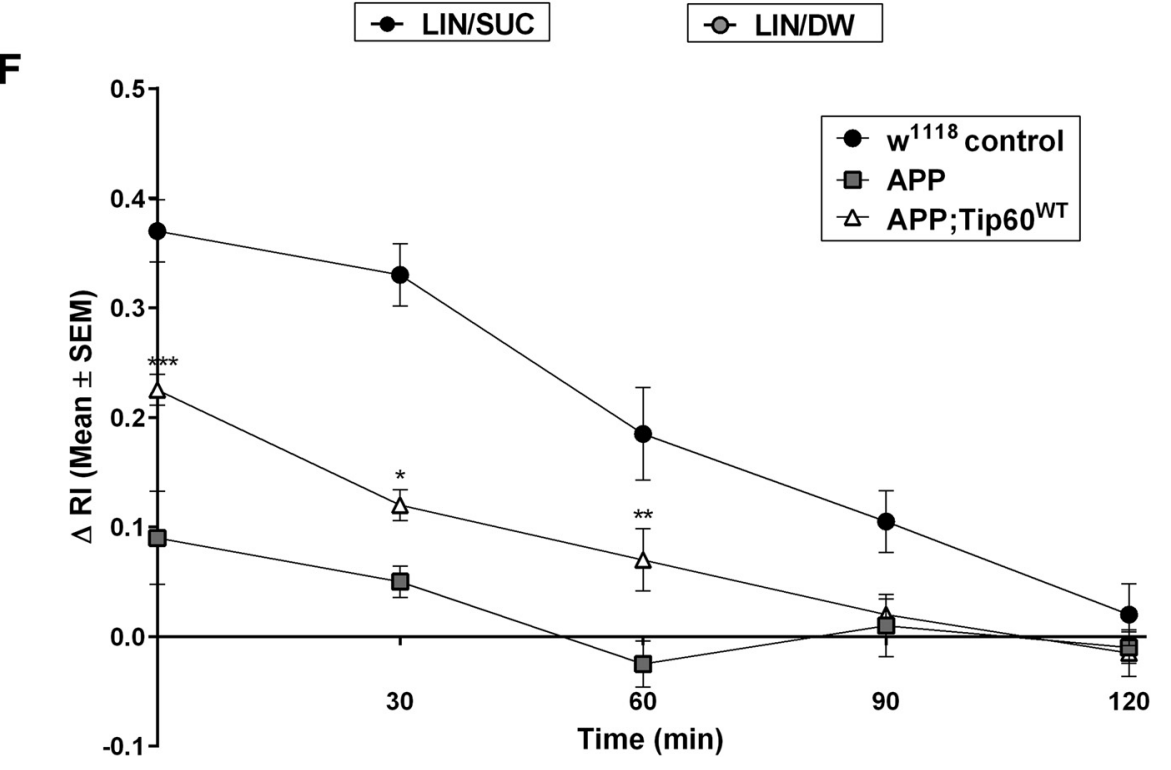

Figure 5. Tip60 HAT improves learning and memory performance in APP-neurodegenerative Drosophila larvae. $A$, Olfactory training and test plates for larval associative learning. For training, third instar larvae were placed on $2.5 \%$ agar plates with a thin layer for $1 \mathrm{~m} \mathrm{SUC} \mathrm{or} \mathrm{DW} \mathrm{with} \mathrm{odorant} \mathrm{(LIN)} \mathrm{spotted} \mathrm{on} \mathrm{a} \mathrm{filter} \mathrm{disk} \mathrm{on} \mathrm{the} \mathrm{lid.} \mathrm{After} 30$ min of training, $50-100$ larvae were transferred to the test plate. After $3 \mathrm{~min}$, the number of larvae that moved in the semicircular area was counted to determine the RI. B, Comparisons of larval Rl after LIN/SUC and LIN/DW (control) conditioning for the indicated genotypes under the elav ${ }^{\mathrm{C} 155}$ pan-neuronal Gal 4 driver indicating associative learning. Statistical significance was calculated using unpaired Student's test. $\boldsymbol{C}-\boldsymbol{E}$, Temporal changes of larval olfactory response after LIN/SUC and LIN/DW (control) conditioning for w ${ }^{1118}(\boldsymbol{C})$, APP (D), and APP;Tip60 ${ }^{\text {WT }}$ (E). Statistical significance was calculated using two-way RM-ANOVA with Sidak's post hoc analysis. $\boldsymbol{F}$, Olfactory memory performances for indicated genotypes plotted in $\Delta R \mathrm{Rl} . \Delta \mathrm{RI}$ was calculated as the difference in RI of LIN/SUC and control LIN/DW ( $n=200)$. Statistical significance was calculated using two-way RM-ANOVA with Tukey's post hoc analysis for comparing APP and APP;Tip60 WT. RIs of all the genotype were normalized using their respective locomotion speed for all experiments. ${ }^{*} p<0.05,{ }^{* *} p<0.01,{ }^{* * *} p<0.001,{ }^{* * *} p<0.0001$. Error bars indicate SEM.

Neuroplasticity gene expression in the brain is repressed during the early stages of $\mathrm{AD}$ neurodegenerative progression and is restored by increasing Tip60

Transcriptional dysregulation of neuroplasticity genes in the brain is a key step in AD etiology (Blalock et al., 2004; Zhang et al., 2013). However, the molecular mechanisms that underlie such gene alterations and when they occur during early $\mathrm{AD}$ progression remain to be determined. Because histone acetylation homeostasis in the brain is critical for transcriptional control of neural genes, we hypothesized that the impairment in Tip60
HAT/HDAC2 balance that we observed in APP larval heads leads to concomitant disruption of neural gene expression in this early $\mathrm{AD}$ stage. To test this hypothesis, we assessed mRNA levels of 15 neuroplasticity genes in the larval brains of two distinct yet synergistic AD Drosophila models: the APP Drosophila model and an $\mathrm{A} \beta_{42}$ Drosophila model specifically designed to induce neural specific $\mathrm{A} \beta_{42}$ expression to increase plaque burden (Iijima and Iijima-Ando, 2008) that occurs only in the aged adult fly brain. The candidate neuroplasticity genes tested were selected because they each have well characterized human homologs, were identi- 

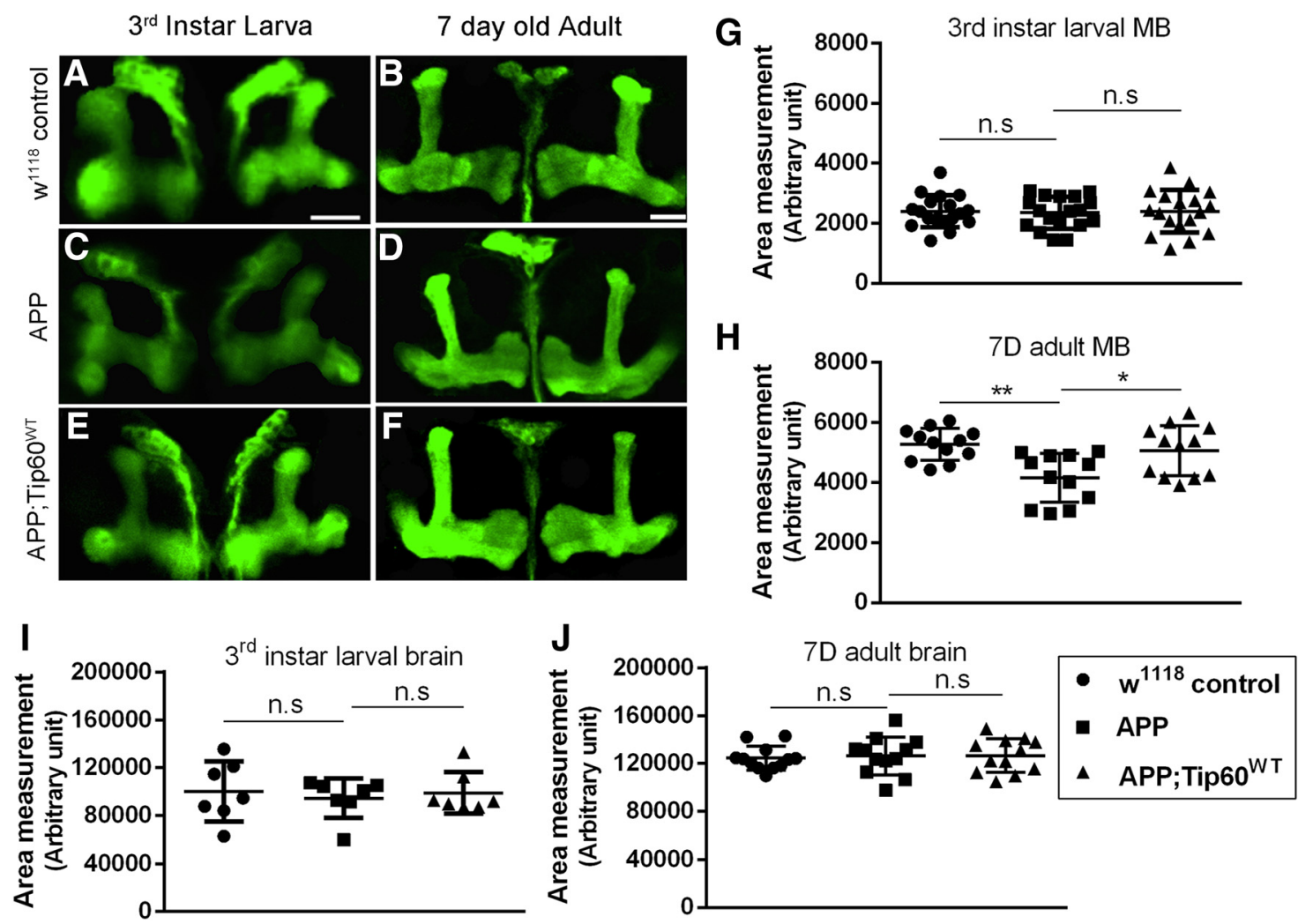

Figure 6. Increasing Tip60 HAT levels rescues MB morphology under APP-neurodegenerative conditions. $\boldsymbol{A}-\boldsymbol{F}$, Representative confocal images of larval and adult MB visualized by $m$ CD8-GFP and stained with anti-GFP to delineate MB from staged third instar larval brain and 7-day old adult brain expressing indicated transgene driven by GFP;0K107-Gal4. Scale bar, $50 \mu$ m. G, H, Quantification of MB area for indicated genotype from larvae $(n=18)$ and adult flies $(n=12)$. One-way ANOVA with Tukey's post hoc analysis was used to determine statistical significance between different genotypes within larval and adult MB. I,, , Quantification of total brain area for indicated genotype from larvae $(n=7)$ and adult flies $(n=12)$ stained with anti-elav and anti-HRP. One-way ANOVA with Tukey's post hoc analysis was used to determine the statistical significance between different genotypes within larval and adult brain. ${ }^{*} p<0.05$, ${ }^{* *} p<0.01$. Error bars indicate SEM.

fied as Tip60 direct target genes in our prior Tip60 ChIP-Seq analysis (Xu et al., 2014), and all play critical roles in cognitive function. RT-qPCR analysis was performed using staged third instar larval brains expressing either APP or $\mathrm{A} \beta_{42}$ under the control of the elav-Gal4 pan-specific neuronal driver. Importantly, these larvae have yet to display amyloid plaque formation and model preclinical defects of the disease (Torroja et al., 1999; Pirooznia et al., 2012b; Johnson et al., 2013). We found that 11 genes tested were significantly repressed in the AD-associated APP larval brains and that 7 of 15 genes were significantly repressed in the $\mathrm{A} \beta_{42}$ larval brains (Fig. $2 A$ ). Genes that were significantly downregulated in both the AD models included those with established roles in synaptic plasticity such as $d s h$ (dishevelled) $\left(F_{(2,6)}=27.17, p=0.0010\right.$, one-way ANOVA with Tukey's post hoc analysis), which is also a key component of the Wntsignaling pathway that has been causatively associated with $\mathrm{AD}$ when disrupted (Cook et al., 1996; Packard et al., 2002; Miech et al., 2008). Additional repressed genes were those with confirmed roles in cognition, such as $\operatorname{sh}$ (shaker) $\left(F_{(2,6)}=18.40, p=0.0028\right.$, one-way ANOVA with Tukey's post hoc analysis), an ion channel involved in activity-dependent synaptic plasticity (Jan et al., $1985)$; futsch $\left(F_{(2,6)}=16.63, p=0.0036\right.$, one-way ANOVA with Tukey's post hoc analysis), a microtubule-associated protein essential in promoting bouton formation by stabilization of MT hairpin loop (Roos et al., 2000; Miech et al., 2008); and $d l g\left(F_{(2,6)}=\right.$ $50.13, p=0.0002$, one-way ANOVA with Tukey's post hoc analysis), a scaffold protein essential for proper synapse formation (Budnik et al., 1996). Repressed genes also included those with well characterized roles in memory and axon extension, such as rut $\left(F_{(2,6)}=7.585, p=0.0228\right.$, one-way ANOVA with Tukey's post hoc analysis) and cadn2 $\left(F_{(2,6)}=28.15, p=0.0009\right.$, one-way ANOVA with Tukey's post hoc analysis) and the dendrite morphogenesis gene chinmo $\left(F_{(2,6)}=14.80, p=0.0048\right.$, one-way ANOVA with Tukey's post hoc analysis). Importantly, mRNA for two neuroplasticity genes (ben and for) were not downregulated in response to either APP or $\mathrm{A} \beta_{42}$ expression, demonstrating specificity of gene repression and our results.

Our previous Tip60 transcriptional profiling and ChIP-Seq findings demonstrated that gene control is a key mechanism by which Tip60 exerts its action in restoring function in a variety of neuronal processes impaired under AD neurodegenerative conditions. Therefore, we hypothesized that Tip60 exerts such neuroprotective action by epigenetically reprogramming gene sets that together promote cognitive function. To test this hypothesis, we performed gene expression analysis on the same 15 Tip60 neuroplasticity genes, this time using staged third instar larval brains expressing APP;Tip60 ${ }^{\mathrm{WT}}$ under the control of the neural elav-Gal4 to increase Tip60 levels in the brain during early APPneurodegenerative progression. For these and the remainder of studies presented, we opted to use the AD-associated APP Drosophila model because we and others have shown that this extremely well characterized model effectively displays both preclinical and late stages of the disease (Torroja et al., 1999; Bolkan et al., 2012; Pirooznia et al., 2012b; Johnson et al., 2013). Remarkably, we found that expression of 9 of the 11 genes that were repressed in the APP larval brain were fully restored by increasing Tip60 HAT levels in the brain of APP;Tip60 ${ }^{\text {WT }}$ larvae (Fig. 2B). Co-immunostaining larval brains from each genotype 

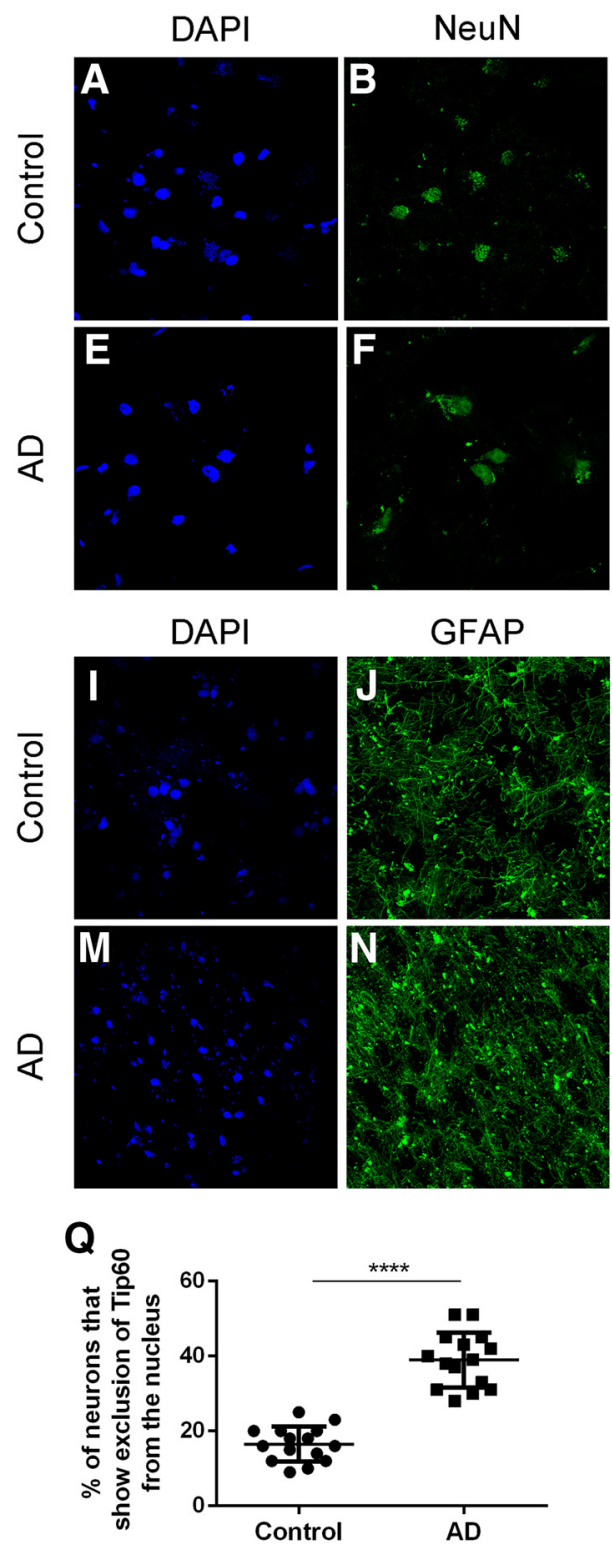

S

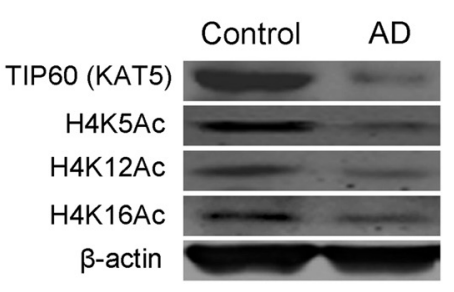

Tip60

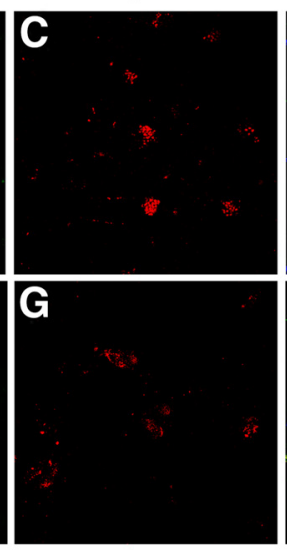

Tip60
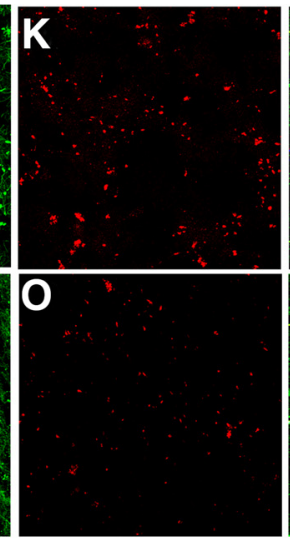

R
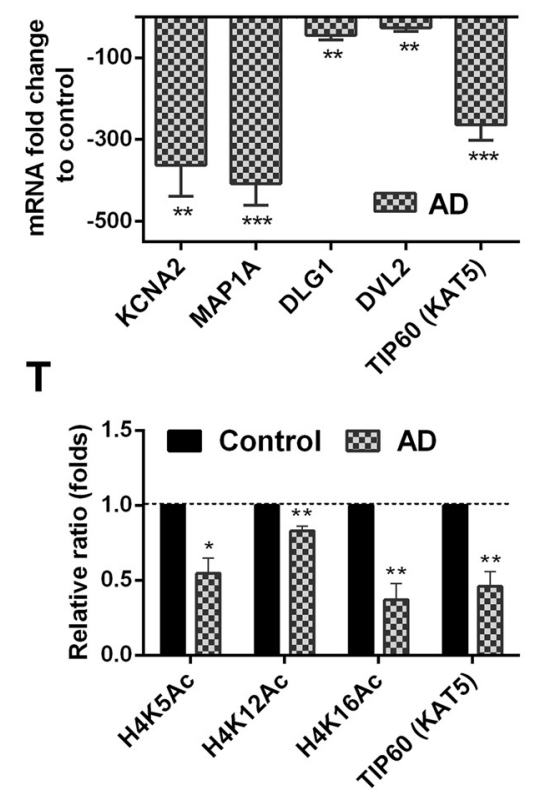

Figure 7. Tip60 HAT function is impaired in human AD hippocampus. Shown are representative confocal images of normal control $(\boldsymbol{A}-\boldsymbol{D})$ and AD (E-H) hippocampal sections double-stained for Tip60 (red) and NeuN (green) with DAPI (blue). Representative confocal images of normal control $(\boldsymbol{I}-\boldsymbol{L})$ and $A D(\boldsymbol{M}-\boldsymbol{P})$ hippocampal sections were double-stained for Tip60 (red) and GFAP (green) with DAPI (blue). Five hippocampal sections from three brains for each healthy control and AD patient were analyzed. Scale bar: $20 \mu \mathrm{m}$. Thin arrows in $\boldsymbol{D}$ and $\boldsymbol{L}$ indicate colocalization of DAPI, Tip60, and NeuN or GFAP, respectively (white). Thick arrows in $\boldsymbol{H}$ and $\boldsymbol{P}$ indicate colocalization of Tip60 and NeuN or GFAP, respectively (yellow). $\mathbf{Q}$, Quantitation of $\boldsymbol{D}$ and $\boldsymbol{H}$ representing the percentage of neurons showing exclusion of Tip 60 from the nucleus. Each dot represents one hippocampal section. 25-30 neurons were scored per hippocampal section for control and AD patients. Five hippocampal sections from three brains for each healthy control and AD patient were analyzed. $R, R T-q P C R$

with antibodies to pan neuronal markers HRP and elav revealed no significant difference in total brain area (Fig. 6I,J), ensuring that the gene changes that we identified in the APP brain were not due to developmental defects affecting brain size. Together, our findings suggest that neuroplasticity gene alterations are an early initial event in $\mathrm{AD}$ possibly caused by Tip60 HAT/HDAC2 disruption and support a transcriptional regulatory role for Tip60 in cognitive restoration in the neurodegenerative brain.

Tip60 reduces repressor HDAC2 recruitment and restores specific epigenetic signatures at neuroplasticity genes in the early neurodegenerative APP larval brain

Thus far, we had observed that Tip60 HAT/HDAC2 balance and concomitant neuroplasticity gene expression is disrupted in the APP larval brain and is restored by increasing Tip60. Therefore, we hypothesized that HDAC2 is inappropriately recruited to Tip60 neuroplasticity genes and that increasing Tip60 levels displaces HDAC2 binding to restore Tip60 mediated gene expression. To test our hypothesis, we selected four Tip60 epigenetically controlled neuroplasticity target genes the we identified in the larval brain, $s h, d l g, f u t s c h$, and $d s h$, the expression of which was repressed in the $\mathrm{AD}$ larval brain and restored by increasing Tip60 for further mechanistic analysis (Fig. 3B). We first investigated whether these genes were bona fide direct targets of Tip60 in the larval brain and if Tip60 recruitment to these gene loci was dependent upon Tip60 HAT activity. To address these questions, we performed a Tip60 ChIP-qPCR assay using chromatin isolated from the larval anterior head region of wild-type control flies $\left(\mathrm{w}^{1118}\right)$ and larvae expressing a dominant-negative Tip60 HAT defective

$$
\leftarrow
$$

was performed on CDNA isolated from normal and AD hippocampus ( $n=3$ brains). Histogram represents relative fold change in mRNA expression level of neuroplasticity genes in the AD hippocampus relative to normal control hippocampus. RT-qPCR for each biological sample was performed in triplicate and the fold change was calculated using the $\Delta \Delta \mathrm{Ct}$ method with GAPDH as a control. S, Representative immunoblot showing Tip60, H4K5ac, H4K12ac, and H4K16ac levels in normal and AD hippocampus ( $n=3$ brains). $\boldsymbol{T}$, Histogram representing densitometer quantification using data from three Western experiments for each normal control and AD hippocampal sample shown in $\boldsymbol{S}$. Statistical significance for all experiments was calculated using unpaired Student's $t$ test. ${ }^{*} p<0.05$, ${ }^{* *} p<0.01,{ }^{* * *} p<0.001,{ }^{* * * *} p<0.0001$. Error bars indicate SEM. 
construct (Tip60 ${ }^{\mathrm{E} 431 \mathrm{Q})}$ that induces Tip60 HAT loss (Lorbeck et al., 2011) under the control of elav-Gal4 pan neuronal driver. This analysis revealed that Tip60 is significantly enriched at each of the four neuroplasticity gene loci in the $\mathrm{w}^{1118}$ control flies (Fig. $3 A ; s h, t_{(4)}=7.656, p=0.0016$, unpaired $t$ test; $d l g, t_{(4)}=5.281$, $p=0.0062$, unpaired $t$ test; futsch, $t_{(4)}=11.16, p=0.0004$, unpaired $t$ test; and $d s h, t_{(4)}=7.442, p=0.0017$, unpaired $t$ test), thus identifying these genes as novel Tip60 gene targets. In direct contrast, dTip60 ${ }^{\mathrm{E} 431 \mathrm{Q}}$ larvae did not show significant Tip60 binding at any of the candidate gene loci (Fig. $3 \mathrm{~A}$ ), suggesting that Tip60 HAT activity is required for its recruitment to these gene loci. No enrichment was observed at the $w g$ gene or at a control repressed heterochromatin (hc) region devoid of gene loci, demonstrating the specificity of our results. We next performed ChIP analysis using Abs against Tip60 or Rpd3 (HDAC2) to assess their enrichment at these four synaptic plasticity gene loci in APP versus APP;Tip60 ${ }^{\text {WT }}$ larval heads under the control of elav-Gal4 driver. Remarkably, Tip60 binding affinity was reduced at all 4 gene loci (Fig. $3 C$; sh, $F_{(2,6)}=42.01, p=0.0003$, one-way ANOVA with Tukey's post hoc analysis; futsch, $F_{(2,6)}=32.16, p=$ 0.0006, one-way ANOVA with Tukey's post hoc analysis; $d s h$, $F_{(2,6)}=77.15, p=0.0001$, one-way ANOVA with Tukey's post hoc analysis; and $d l g, F_{(2,6)}=44.26, p=0.0003$, one-way ANOVA with Tukey's post hoc analysis) in APP larval heads and was restored by increasing Tip60 levels in the APP;Tip60 ${ }^{\text {WT }}$ larval heads. Conversely, Rpd3(HDAC2) binding was significantly enriched at all of these 4 Tip60 neuroplasticity genes in AD larval heads and reduced almost back to wild-type levels at two of these genes (Fig. 3D; futsch, $F_{(2,6)}=38.21, p=0.0004$, one-way ANOVA with Tukey's post hoc analysis; dsh, $F_{(2,6)}=28.58, p=$ 0.0009 , one-way ANOVA with Tukey's post hoc analysis) by increasing Tip60 levels. As expected, there was enrichment of Rpd3 (HDAC2) at the control repressed heterochromatic region in all three genotypes ( $\mathrm{w}^{1118}$, APP, and APP;Tip60 ${ }^{\mathrm{WT}}$ ), which is consistent with Rpd3's role in repression.

In vitro studies have shown that Tip60 has specificity in acetylating lysine-specific histone residues $\mathrm{H} 3 \mathrm{~K} 9, \mathrm{H} 4 \mathrm{~K} 12$, and $\mathrm{H} 4 \mathrm{~K} 16$, all of which play established roles in learning and memory (Fischer et al., 2007) by controlling gene expression via chromatin packaging in neurons. To test whether Tip60 acetylates these residues at these specific neuroplasticity genes in the larval brain in vivo, we performed ChIP-qPCR using chromatin isolated from larval heads to assess enrichment levels of $\mathrm{H} 3 \mathrm{~K} 9 \mathrm{ac}, \mathrm{H} 4 \mathrm{~K} 12 \mathrm{ac}$, and $\mathrm{H} 4 \mathrm{~K} 16 \mathrm{ac}$ at each of the target gene loci in $\mathrm{w}^{1118}$ and Tip60 HAT mutant Tip $60^{\mathrm{E} 431 \mathrm{Q}}$ larvae. We found that each of the genes was enriched for H4K12ac (Fig. 4A) and H4K16ac (Fig. 4B) in wildtype $\mathrm{w}^{1118}$ larvae. In direct contrast, no significant enrichment was observed in the HAT mutant Tip $60{ }^{\mathrm{E} 431 \mathrm{Q}}$ larvae except at the sh gene loci, where enrichment levels for $\mathrm{H} 4 \mathrm{~K} 12 \mathrm{ac}$ were higher than that in wild-type $\mathrm{w}^{1118}$ larvae (Fig. 4A). We observed no significant change in $\mathrm{H} 3 \mathrm{~K} 9$ acetylation enrichment levels in either the $\mathrm{w}^{1118}$ or the Tip $60^{\mathrm{E} 431 \mathrm{Q}}$ mutant (Fig. 4C), suggesting that H3K9 may not serve as a target lysine residue for Tip60 in the brain at these specific neuroplasticity genes. We next hypothesized that Tip60-mediated histone acetylation at the four synaptic plasticity gene loci $(s h, d l g, f u t s c h$, and $d s h)$ would be reduced in the AD larval brain and restored by increasing Tip60 HAT levels. To test this hypothesis, we used ChIP to assess acetylation enrichment at these synaptic plasticity gene loci using Abs to acetylated (Ac) histone marks AcH4K5, 12, and 16 because we found that these marks are generated by Tip60 HAT action in the larval brain (Fig. 4A, B). Our results showed that Ac levels at each of these marks at the 4 Tip60 synaptic plasticity gene target loci are signif- icantly reduced in the $\mathrm{AD}$ larval brain and restored by increasing Tip60 HAT levels in the brain (Fig. 4D-F). Together, our findings support a model by which increasing Tip60 in the AD larval brain restores Tip60 HAT binding and acetylation levels at synaptic plasticity genes by reducing both Rpd3 (HDAC2) expression levels and inappropriate protein recruitment, thus restoring Tip60mediated synaptic plasticity gene expression.

To confirm mammalian conservation of these neuroepigenetic signatures, ChIP was performed on hippocampal tissues dissected from mixed equal numbers of male and female 6-month-old C57BL wild-type mice at the four mouse homolog gene loci using Abs against Tip60 and acetylation mark H4K16. Our results showed that the Tip60-mediated neuroepigenetic signatures that we identified at select neuroplasticity genes in the larval head are entirely recapitulated in mouse hippocampus, but not in negative liver tissue specificity control (Fig. $4 G ; K c n a 2, t_{(10)}=$ 33.14, $p=0.0001$, unpaired $t$ test; Map1a, $t_{(10)}=4.073, p=$ 0.0022 , unpaired $t$ test; $D \lg 1, t_{(10)}=2.925, p=0.0152$, unpaired $t$ test; $D v l 2, t_{(10)}=16.05, p=0.0001$, unpaired $t$ test; Fig. $4 H$; Kcna2, $t_{(10)}=19.74, p=0.0001$, unpaired $t$ test; Map1a, $t_{(10)}=$ $4.355, p=0.0014$, unpaired $t$ test $D \lg 1, t_{(10)}=3.265, p=0.0085$, unpaired $t$ test; and $D v l 2, t_{(10)}=3.767, p=0.0037$, unpaired $t$ test). Together, our results identify novel Tip60 HAT-mediated epigenetic mechanisms underlying the transcriptional control of $s h, d l g, f u t s c h$, and $d s h$ in the larval brain in vivo and show that these epigenetic neural gene control mechanisms are conserved in mammalian hippocampus

\section{Tip60 HAT action improves learning and memory deficits in APP neurodegenerative larvae}

Our previous findings demonstrated that increased Tip60 HAT levels suppresses early defects in axonal outgrowth and transport in the $\mathrm{AD}$ brain and restores associated complex behavioral deficits that include disruption of sleep/wake cycles and locomotor ability (Pirooznia et al., 2012a; Johnson et al., 2013). Moreover, we also demonstrated that defects in learning and memory in the AD adult fly is alleviated by increasing Tip60 HAT levels in the brain (Xu et al., 2014). However, whether such cognitive deficits also occur early during $\mathrm{AD}$-associated neurodegenerative progression and if they can be prevented by increasing Tip60 HAT levels in the brain remained to be determined. To address these questions, we performed a single odor paradigm for olfactory associative learning (Honjo and Furukubo-Tokunaga, 2005; Fig. $5 A$ ) using APP versus APP;Tip60 ${ }^{\text {WT }}$ Drosophila third instar larvae under the control of pan-neuronal elav-Gal4 driver. The larvae were conditioned to associate a given odor, LIN, to an appetitive gustatory reinforcer, SUC. These larvae were exposed to LIN for $30 \mathrm{~min}$ in association with $1 \mathrm{~m} \mathrm{SUC} \mathrm{on} \mathrm{an} \mathrm{agar} \mathrm{plate.}$ After conditioning, the larvae were tested for olfactory response on the test plate (Fig 5A). Importantly, the higher RIs of all of the genotype were normalized using their respective locomotion speed. We observed an enhanced migration of the LIN/SUC conditioned $\mathrm{w}^{1118}$ larvae to the odor (LIN) with significantly higher RI than control larvae that had been exposed to LIN in conjunction with DW (Fig. $5 B ; t_{(4)}=8.025, p=0.0013$, unpaired $t$ test). In direct contrast, the APP larvae showed no significant difference in migration toward the odor between larvae trained with LIN/SUC compared with the control larvae (LIN/DW), indicating that APP larvae showed reduced initial learning because they could not learn to associate the odor (LIN) with an appetitive stimulus (SUC) (Fig. $5 B ; t_{(4)}=2.393, p=0.075$, unpaired $t$ test). We next investigated whether increasing Tip60 HAT levels could rescue the learning deficits observed in the APP larvae. Remark- 
Table 1. Sensory acuities

\begin{tabular}{lll}
\hline Genotype & Olfactory response (LIN) & Gustatory response (1 m SUC) \\
\hline W $^{1118}$ & $0.35 \pm 0.06$ & $0.33 \pm 0.04$ \\
APP & $0.44 \pm 0.01$ & $0.35 \pm 0.06$ \\
APP;Tip60 WT & $0.40 \pm 0.01$ & $0.30 \pm 0.05$ \\
\hline
\end{tabular}

Olfactory and gustatory responses were examined as described in the Materials and Methods. Data was obtained from at least four independent experiments. No significant differences between olfactory or gustatory response were observed between any of the genotypes. Results are shown as \pm SEM.

ably, APP;Tip60 ${ }^{\text {WT }}$ larvae exhibited an RI increment with associative LIN/SUC training compared with control LIN/DW that was almost comparable to the $\mathrm{w}^{1118}$ control, indicating the Tip60 mediated rescue of learning deficits under APP-induced neurodegenerative conditions (Fig. $5 B ; t_{(4)}=4.065, p=0.0153$, unpaired $t$ test).

The following multiple phases of memory acquisition and consolidation have been identified in Drosophila (Tully et al., 1994): short term memory (STM), medium-term memory (MTM), anesthesia-resistant memory (ARM), and long-term memory (LTM). To study early phase memory, we compared temporal changes of the larval response after LIN/SUC conditioning for up to $120 \mathrm{~min}$. Larval olfactory response after the LIN/SUC conditioning in the $\mathrm{w}^{1118}$ larvae went down to almost control (LIN/DW) levels after $120 \mathrm{~min}$ (Fig. $5 C ; F_{(4,8)}=9.042$, $p=0.0046$, two-way RM-ANOVA with Sidak's post hoc analysis). RI comparison between LIN/SUC and LIN/DW showed significant memory performance $(\Delta \mathrm{RI})$, which was retained until 120 min (Fig. $5 F$ ). Because APP larvae still exhibited some residual learning capability, we next investigated whether they were able to remember the little they could learn. Olfactory response of APP larvae after LIN/SUC conditioning did not show a significant difference compared with control (LIN/DW) at 0 and 30 $\mathrm{min}$ and was the same as control (LIN/DW) by $60 \mathrm{~min}$ (Fig. 5D; $F_{(4,8)}=2.298, p=0.1471$, two-way RM-ANOVA with Sidak's post hoc analysis). Accordingly, these APP larvae showed significant impairment in MTM performance $(\Delta \mathrm{RI})$, where memory trace became undetectable by $60 \mathrm{~min}$ (Fig. $5 F$ ). Olfactory and gustatory responses of APP larvae were normal compared with $\mathrm{w}^{1118}$ control larvae (Table 1$)$. We next investigated whether increasing Tip60 HAT activity could restore medium phase memory impairment in the APP larvae. Remarkably, olfactory response of the APP;Tip60 ${ }^{\mathrm{WT}}$ after LIN/SUC conditioning was significantly higher at 0 and $30 \mathrm{~min}$ compared with control (LIN/ DW) and gradually decreased to control (LIN/DW) level by 90 $\min$ (Fig. $5 E ; F_{(4,8)}=19.87, p=0.0003$, two-way RM-ANOVA with Sidak's post hoc analysis). Accordingly, RI comparison between LIN/SUC and LIN/DW showed significant memory performance $(\Delta \mathrm{RI})$, which was retained beyond $60 \mathrm{~min}$ and gradually lost by $90 \mathrm{~min}$, indicating partial rescue of APPinduced memory deficits (Fig. $5 F ; F_{(8,12)}=8.279, p=0.0007$, two-way ANOVA with Tukey's post hoc analysis). The olfactory and gustatory responses of APP;Tip60 ${ }^{\text {WT }}$ larvae were normal compared with $\mathrm{w}^{1118}$ control larvae (Table 1). Together, these findings demonstrate that defects in olfactory-associative appetitive learning and memory retention occur early during ADassociated neurodegenerative progression and can be improved by increasing Tip60 HAT levels.

\section{Increasing Tip60 HAT levels rescues MB morphology under APP-induced neurodegenerative conditions}

Our prior studies demonstrated that the HAT Tip60 is produced robustly in the fly brain and MBs (Xu et al., 2014), which control olfactory function (Heisenberg, 2003; Gerber et al., 2004), and is required for both $\mathrm{MB}$ axonal outgrowth and learning and memory (Xu et al., 2014). Accordingly, our data here show that increasing Tip60 in the larval brain improves APP-mediated impairments in olfactory learning and memory that occur early during neurodegenerative progression (Fig. $5 B, F$ ). Therefore, we investigated whether the early cognitive deficits that we observed in APP larvae were accompanied by structural defects in the MBs and if these defects could be rescued by increasing Tip60 levels. To address these questions, we used the MB GAL4 driver OK107, which expresses GAL4 in discrete neuronal populations in the adult fly brain, including robust expression in the Kenyon cells, the intrinsic neurons of the $\mathrm{MB}$, as well as lower expression in the pars intercerebralis, suboesophageal ganglion, and optic lobes. We crossed flies carrying a UAS-mCD8-GFP marker in conjunction with the MB-specific OK107-GAL4 driver to control $\mathrm{w}^{1118}$, APP, or APP;Tip60 ${ }^{\mathrm{WT}}$ fly lines to mark MB neurons with GFP for visualization. MBs from dissected brains from either staged third instar larvae or aged 7-d-old adult flies for each genotype were stained with antibodies to GFP to delineate the MB and $\mathrm{MB}$ area was quantitated for each genotype. Quantitation analysis showed no observable defects in $\mathrm{MB}$ morphology in the larval AD brains (Fig. 6A, C,E, $G ; F_{(2,51)}=0.03998, p=0.9608$, one-way ANOVA with Tukey's post hoc analysis). In contrast, we observed an overall reduction in $\mathrm{MB}$ total area in 7-d-old $\mathrm{AD}$ flies compared with control $\mathrm{w}^{1118}$ flies (Fig. $6 \mathrm{~B}, \mathrm{D}, \mathrm{H} ; \mathrm{F}_{(2,33)}=7.699$, $p=0.0018$, one-way ANOVA with Tukey's post hoc analysis), indicative of axonal outgrowth impairment (Xu et al., 2014). This reduction only appeared in approximately half of the MBs that we assessed, indicating partial penetrance. In addition, we also coimmunostained staged third instar larval brains and 7-d-old adult brains expressing APP or APP;Tip $60^{\mathrm{WT}}$ under the control of the pan neural elav-Gal4 driver with antibodies to the panneuronal markers HRP and elav. We observed no significant difference in total brain area (Fig. $6 I ; F_{(2,18)}=0.1528, p=0.8594$, one-way ANOVA with Tukey's post hoc analysis, Figure 6J; $F_{(2,33)}=$ $0.08751, p=0.9164$, one-way ANOVA with Tukey's post hoc analysis), indicating that the $\mathrm{MB}$ size reduction that we observed in the APP brain was not due to APP-induced developmental defects affecting overall brain size. Importantly, we found that this $\mathrm{AD}$-associated axonal outgrowth phenotype was effectively rescued in the 7-d-old adult by increasing Tip60 HAT levels in the MBs (Fig. 6F, H). Together, our results show that, whereas APPinduced neurodegeneration in the MBs causes learning and memory deficits in flies as young as third instar larvae, broad MB morphological defects are not consistently observable until adult flies are aged to day 7.

\section{Tip60 HAT function is impaired in human AD hippocampus}

To confirm human AD disease relevance, we next investigated whether the Tip60 neuroplasticity genes that we identified using fly and mouse models are epigenetically misregulated in the human AD brain due to loss of Tip60 HAT function. To address this question, we first tested whether Tip60 protein levels are reduced in neurons and glial cells in human $\mathrm{AD}$ hippocampus by performing immunohistochemistry on frozen hippocampal tissue sections from age-matched healthy control and AD hippocampus using antibodies against Tip60 and DAPI to mark neuronal nuclei and co-staining with antibodies to either neuronal marker NeuN or glial marker GFAP. Tip60 was shown to be present in both neurons (Fig. 7A-D) and glial cells (Fig. $7 I-L$ ), as indicated by Tip60 co-staining with NeuN or GFAP, respectively, and was significantly reduced in both cell types in the AD hippocampus (Fig. $7 E-H, M-P$ ) compared with the control hippocampus. Fur- 
ther, whereas we observed Tip60's typical punctate distribution pattern in the nucleus and cytoplasm (Fig. 7D) in neurons from healthy hippocampus tissues, Tip60 was largely but not exclusively excluded from neuronal nuclei in the AD hippocampal tissue (Fig. $7 H, Q ; t_{(28)}=9.967, p=0.0001$, unpaired $t$ test). The regions of the hippocampus assessed were the same in healthy control and AD patients. We next assessed Tip60 protein levels and bulk histone acetylation levels for Tip60-mediated histone Ac marks H4K5, H4K12, and H4K16 using Western blot analysis on whole protein extracts isolated from age-matched healthy control and $\mathrm{AD}$ hippocampus. These studies revealed reduced Tip60 levels and a reduction in the same Tip60-mediated histone Ac marks (H4K5,12,16) that we identified as being significantly reduced in the $\mathrm{AD}$ larval brain (Fig. $7 S, T$; H4K5Ac, $t_{(4)}=4.534$, $p=0.0105$, unpaired $t$ test; H4K12Ac, $t_{(4)}=5.645, p=0.0048$, unpaired $t$ test; H4K16Ac, $t_{(4)}=5.869, p=0.0042$, unpaired $t$ test; KAT5, $t_{(4)}=5.544, p=0.0052$, unpaired $t$ test). To test whether Tip60-mediated transcriptional control of synaptic plasticity genes is compromised in the AD patient's brain, we performed RT-qPCR on cDNA isolated from age-matched, healthy control and AD patient hippocampal tissues on the four Tip60 human homolog neuroplasticity genes for which expression, Tip60 binding, and acetylation were reduced in the APP fly brain and restored by increased Tip60. Our results demonstrated that expression of all 4 genes and Tip60 mRNA levels were significantly reduced in the human $\mathrm{AD}$ hippocampus compared with healthy controls (Fig. $7 R$; KCNA2, $t_{(4)}=8.186, p=0.0012$, unpaired $t$ test; MAP1A, $t_{(4)}=13.25, p=0.0002$, unpaired $t$ test; $D L G 1, t_{(4)}=6.995, p=0.0022$, unpaired $t$ test; DVL2, $t_{(4)}=$ $5.498, p=0.0053$, unpaired $t$ test; KAT5, $t_{(4)}=11.72, p=0.0003$, unpaired $t$ test), similar to what we observed in the AD-associated APP larval brain (Fig. 2A). Together, our results show that Tip60 HAT function in epigenetic neuroplasticity gene control is compromised in both the early $\mathrm{AD}$ neurodegenerative larval brain and in both neural and glial cell types in the aged human $\mathrm{AD}$ hippocampus.

\section{Discussion}

\section{Disruption of Tip60 HAT/HDAC2 homeostasis and} concomitant repression of neuroplasticity genes in the brain is an early event in $\mathrm{AD}$ neurodegenerative progression

Disruption of such HAT/HDAC balance is believed to cause epigenetically mediated transcriptional dysregulation that negatively affects cellular homeostasis and cognitive function (Langley et al., 2005). Indeed, reduced histone acetylation levels are found in the brains of animal models for multiple types of progressive cognitive disorders, including AD. Accordingly, Gräff et al. (2012) reported elevated levels of class I HDAC2 mRNA and protein and enhanced enrichment of HDAC2 at synaptic plasticity genes in the brains from their CK-p25 AD mouse model and increased levels of HDAC2 in the hippocampus of AD patients. Despite these important findings, such studies investigating epigenetic alterations in $\mathrm{AD}$ to date have conventionally been performed using brains that already displayed amyloid plaque accumulation. Therefore, whether such changes occur early during disease progression remained unknown. In this study, we demonstrate that elevated Rpd3(HDAC2) mRNA and protein levels and inappropriate enrichment of Rpd3(HDAC2) at certain neuroplasticity genes occur in the early neurodegenerative APP larval brain well before amyloid plaque formation and adult lethality occurs. Class I HDACs are divided into an HDAC1/2 subclass that comprises the vertebrate HDAC1 and HDAC2 proteins and Drosophila Rpd 3 that is the sole HDAC1/2 ortholog in the fly
(Gregoretti et al., 2004). It is well documented that HDAC2 and Rpd3 play similar roles in neural function in that they have both been implicated in memory formation and synaptic activity in mouse and Drosophila, respectively (Guan et al., 2009; Tea et al., 2010; Fitzsimons and Scott, 2011). Further, Gräff et al. (2012) reported elevated levels of HDAC2 mRNA and protein, but not HDAC1, in the brains from their CK-p25 AD mouse model and human $\mathrm{AD}$ hippocampus. Therefore, our results strongly suggest that the effect of APP in repression of plasticity genes in Drosophila is highly dependent upon elevated Rpd3 due to its high homology and functional redundancy with mammalian HDAC2. Nevertheless, we do not rule out the possibility that some APP phenotypic effects may result from Rpd3's homology to both HDAC1 and 2. Our findings are the first to suggest that elevation of HDAC2 levels and inappropriate HDAC2 recruitment to neuroplasticity genes is an early event in $\mathrm{AD}$ neurodegenerative progression.

Transcriptional dysregulation of neuroplasticity genes in the brain is a well characterized key step in AD etiology. However, whether such gene expression alterations are an initial triggering cause for MCI and subsequent $\mathrm{AD}$ pathophysiology or if they are a consequence of $\mathrm{A} \beta$ plaque deposition that then accelerates disease progression remains unclear. Transcriptome studies to date that identify neuroplasticity gene misregulation in brains from postmortem $\mathrm{AD}$ patients have only used brain samples so characterized by $\mathrm{A} \beta$ plaque deposition. Here, we show that the majority of neuroplasticity genes that we tested in the brains of two distinct yet synergistic APP and $\mathrm{A} \beta_{42}$ AD Drosophila models are repressed well before both fly models display adult amyloid plaque formation and lethality. Therefore, we speculate that early transcriptional dysregulation of neuroplasticity genes contributes to the MCI believed to represent a transitional period before full development of AD (Petersen et al., 2009; Minhas et al., 2017). In support of this concept, Leidinger et al. (2013) performed comparative analysis of blood samples from patients with $A D$ versus $M C I$ and found similar alterations in specific miRNAs that target and modulate neuroplasticity gene expression. Further, elegant work by Gjoneska et al. (2015) demonstrated that distinct transcriptome alterations occur in an $\mathrm{AD}$-associated CKp25 mouse model at different developmental stages of disease induction that can be classified as transient (early changes occurring after 2 weeks only), late-onset ( 6 weeks only), and consistent (both) expression classes. Accordingly, we and others show that most of the neuroplasticity genes that we identify as being repressed during the early stages of APP- and $\mathrm{A} \beta_{42}$-induced neurodegeneration in the larval brain are also repressed in postmortem $\mathrm{AD}$ hippocampal tissues from patients that exhibit late stages of the disease (Blalock et al., 2004). Therefore, we propose that some of the neuroplasticity gene alterations that we identify during early $\mathrm{AD}$ progression before amyloid plaque formation persist or possibly worsen during later stages of the disease.

\section{Increasing Tip60 in the AD brain restores Tip60 HAT/HDAC2 balance and reinstates neuroplasticity gene expression, brain morphology, and cognition}

Although findings from our group and others point to elevated HDAC2 levels as an initial trigger for neuroplasticity gene repression that likely contributes to the MCI that patients exhibit before $\mathrm{AD}$ diagnosis, whether there are changes in levels of specific HATs that could act to counterbalance HDAC2 homeostasis in such epigenetic gene control remain unknown. Our previous findings (Pirooznia et al., 2012a; Johnson et al., 2013; Xu et al., 
NEURODEGENERATION

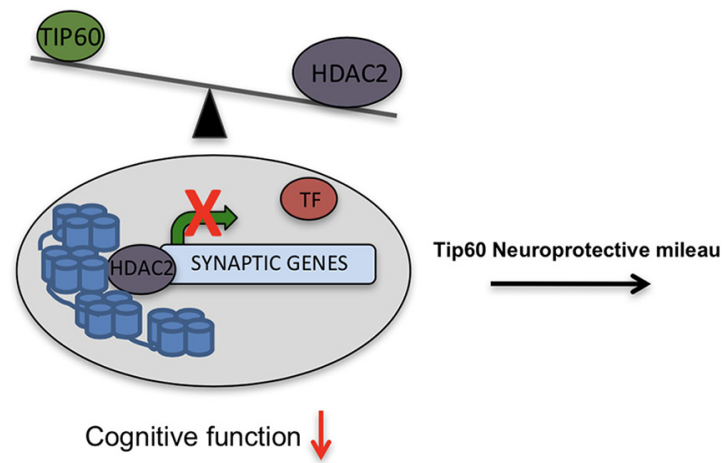

NEUROPROTECTION

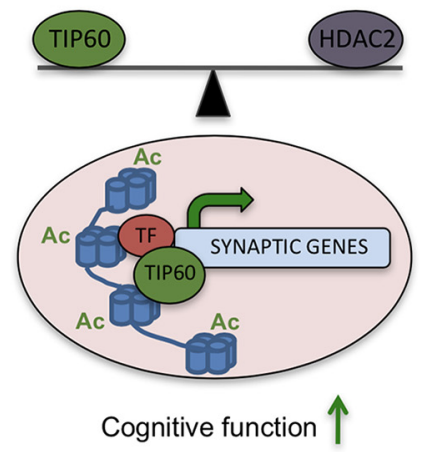

Figure 8. Tip60 promotes neural health by reinstating Tip60 HAT/HDAC2 balance in the AD brain. Our results support a model for $\mathrm{MCl}$ in the preclinical stages of $\mathrm{AD}$ that involves disruption of neuronal acetylation homeostasis in the brain due to loss of Tip60 HAT activity and gain in HDAC2 activity. Such alterations in Tip60 HAT/HDAC2 balance result in inappropriate recruitment of HDAC2 to neuroplasticity genes, resulting in repression of their expression. Increasing cellular levels of specific neural HATs such as Tip60 reinstates acetylation homeostasis in the brain, which restores neuroplasticity gene expression profiles and thus promotes neural health.

2014) support a model by which the HAT Tip60 may act to restore general acetylation homeostasis and, in particular, neuroplasticity gene expression programs during preclinical stages of AD by specifically counterbalancing HDAC2 levels. In support of this concept, here, we show that repressed neuroplasticity genes $s h$, futsch, $d l g$, and $d s h$ in the APP larval brain that have been shown previously to be critical for cognitive neural processes affected in AD (Jan et al., 1985; Budnik et al., 1996; Cook et al., 1996; Roos et al., 2000) show inappropriately enhanced HDAC2 binding and reduced enrichment for Tip60 and histone acetylation well before $\mathrm{A} \beta$ plaque formation and adult lethality occur. Remarkably, increasing Tip60 levels restores appropriate Tip60 HAT/HDAC2 homeostatic balance, at least in part by decreasing HDAC2 levels. Increasing Tip60 levels in the APP larval brain also effectively reverse alterations in neuroepigenetic signatures, relieve repression of neuroplasticity gene expression, and reinstate brain morphology and cognition. Together, our findings identify $s h$, futsch, dlg, and $d s h$ as bona fide direct Tip60 targets in both fly brain and mouse hippocampus and support a model by which disruption of Tip60 HAT/HDAC2 balance and resulting epigenetic repression of neuroplasticity genes is a critical initial step in the $\mathrm{AD}$ pathological process that can be effectively alleviated by increasing Tip60 HAT levels (Fig. 8).

The extent to which bulk acetylation levels are altered in $\mathrm{AD}$ remains controversial. Reduced, elevated, and unchanged basal acetylation levels have all been reported in postmortem $\mathrm{AD}$ human samples (Zhang et al., 2012; Lu et al., 2014; Narayan et al., 2015). Here, we show that reduced bulk protein levels of Tip60 and Tip60 mediated specific histone acetylation marks H4K5, 12, and 16 in human $\mathrm{AD}$ hippocampal tissue. Importantly, histone acetylation at $\mathrm{H} 4 \mathrm{~K} 5,12$, and 16 have each been linked to the control of genes with well established roles in cognitive function (Peixoto and Abel, 2013). Therefore, AD might be more related to disruption of certain HATs such as Tip60 that function to generate histone acetylation patterns at genes specifically associated with certain processes such as learning and memory and these changes are masked in studies that assess bulk levels of histone acetylation. Because we found that these same marks were reduced at Tip60 neuroplasticity gene loci during early APP-mediated neurodegeneration progression in the larval brain, we propose that these alterations are initiated during the preclinical MCI stages of $\mathrm{AD}$ and persist or worsen during disease progression.

\section{Tip60 HAT activators as therapeutics in neurodegenerative disorders}

Pharmacological treatments aimed at increasing histone acetylation by inhibiting HDAC action in these models have shown reversal of cognitive deficits (Langley et al., 2005; Fischer et al., 2010; Gräff et al., 2011). Although promising, many currently used HDAC inhibitors (HDACi's) lack target specificity because they act to increase global levels of acetylation (Haberland et al., 2009; Fischer et al., 2010; Johnson et al., 2013), raising concerns about their applicability. Here, we identify for the first time a specific set of Tip60 neuroplasticity genes in which expression, histone acetylation, and binding affinity for Tip60 is substantially reduced in the APP-neurodegenerative fly brain and restored by increasing Tip60. Further, we show that Tip60 exerts this neuroprotective function by generating site-specific chromatin marks that reinstate neuroplasticity gene expression profiles that are inappropriately repressed in $\mathrm{AD}$ and reinstates brain morphology and function in learning and memory (Rouaux et al., 2003). Our findings strongly support the concept of exploring the efficacy of specific Tip60 HAT activators and identifying and manipulating additional epigenetically misregulated Tip60 target genes to identify those that affect specific AD pathways and associated phenotypes functionally. Such studies would enhance the development of early therapeutic treatments to slow or protect against cognitive decline because they would have more direct and specific effects over more general HDACi treatment (Selvi et al., 2010). Recent exciting advances have been made in the development of two HAT activator molecules, a PCAF activator and a CBP/p300 activator (Chatterjee et al., 2013), both of which can cross the blood-brain barrier and function to enhance memory. Further elucidation of the molecular mechanisms underlying select HATs with neural function, such as Tip60, and their gene target specificity in animals should provide earlier, safer, and more selective ways to promote histone acetylation-mediated cognitive enhancement benefits in the clinical setting.

\section{References}

Blalock EM, Geddes JW, Chen KC, Porter NM, Markesbery WR, Landfield PW (2004) Incipient Alzheimer's disease: microarray correlation analyses reveal major transcriptional and tumor suppressor responses. Proc Natl Acad Sci U S A 101:2173-2178. CrossRef Medline

Bolkan BJ, Triphan T, Kretzschmar D (2012) Beta-secretase cleavage of the fly amyloid precursor protein is required for glial survival. J Neurosci 32:16181-16192. CrossRef Medline

Borrelli E, Nestler EJ, Allis CD, Sassone-Corsi P (2008) Decoding the epigenetic language of neuronal plasticity. Neuron 60:961-974. CrossRef Medline

Budnik V, Koh YH, Guan B, Hartmann B, Hough C, Woods D, Gorczyca M (1996) Regulation of synapse structure and function by the Drosophila tumor suppressor gene dlg. Neuron 17:627-640. CrossRef Medline

Chatterjee S, Mizar P, Cassel R, Neidl R, Selvi BR, Mohankrishna DV, Vedamurthy BM, Schneider A, Bousiges O, Mathis C, Cassel JC, Eswaramoorthy M, Kundu TK, Boutillier AL (2013) A novel activator of CBP/p300 acetyltransferases promotes neurogenesis and extends memory duration in adult mice. J Neurosci 33:10698-10712. CrossRef Medline

Cook D, Fry MJ, Hughes K, Sumathipala R, Woodgett JR, Dale TC (1996) Wingless inactivates glycogen synthase kinase-3 via an intracellular sig- 
nalling pathway which involves a protein kinase C. EMBO J 15:45264536. Medline

Fischer A, Sananbenesi F, Wang X, Dobbin M, Tsai LH (2007) Recovery of learning and memory is associated with chromatin remodelling. Nature 447:178-182. CrossRef Medline

Fischer A, Sananbenesi F, Mungenast A, Tsai LH (2010) Targeting the correct HDAC(s) to treat cognitive disorders. Trends Pharmacol Sci 31:605617. CrossRef Medline

Fitzsimons HL, Scott MJ (2011) Genetic modulation of Rpd3 expression impairs long-term courtship memory in Drosophila. PLoS One 6:e29171. CrossRef Medline

Fossgreen A, Brückner B, Czech C, Masters CL, Beyreuther K, Paro R (1998) Transgenic Drosophila expressing human amyloid precursor protein show gamma-secretase activity and a blistered-wing phenotype. Proc Natl Acad Sci U S A 95:13703-13708. CrossRef Medline

Gerber B, Tanimoto H, Heisenberg M (2004) An engram found? evaluating the evidence from fruit flies. Curr Opin Neurobiol 14:737-744. CrossRef Medline

Gjoneska E, Pfenning AR, Mathys H, Quon G, Kundaje A, Tsai LH, Kellis M (2015) Conserved epigenomic signals in mice and humans reveal immune basis of Alzheimer's disease. Nature 518:365-369. CrossRef Medline

Gräff J, Kim D, Dobbin MM, Tsai LH (2011) Epigenetic regulation of gene expression in physiological and pathological brain processes. Physiol Rev 91:603-649. CrossRef Medline

Gräff J, Rei D, Guan JS, Wang WY, Seo J, Hennig KM, Nieland TJ, Fass DM, Kao PF, Kahn M, Su SC, Samiei A, Joseph N, Haggarty SJ, Delalle I, Tsai LH (2012) An epigenetic blockade of cognitive functions in the neurodegenerating brain. Nature 483:222-226. CrossRef Medline

Greeve I, Kretzschmar D, Tschäpe JA, Beyn A, Brellinger C, Schweizer M, Nitsch RM, Reifegerste R (2004) Age-dependent neurodegenertion and Alzheimer-amyloid plaque formation in transgenic Drosophila. J Neurosci 24:3899-38906. CrossRef Medline

Gregoretti IV, Lee YM, Goodson HV (2004) Molecular evolution of the histone deacetylase family: functional implications of phylogenetic analysis. J Mol Biol 338:17-31. CrossRef Medline

Guan JS, Haggarty SJ, Giacometti E, Dannenberg JH, Joseph N, Gao J, Nieland TJ, Zhou Y, Wang X, Mazitschek R, Bradner JE, DePinho RA, Jaenisch R, Tsai LH (2009) HDAC2 negatively regulates memory formation and synaptic plasticity. Nature 459:55-60. CrossRef Medline

Haberland M, Montgomery RL, Olson EN (2009) The many roles of histone deacetylases in development and physiology: implications for disease and therapy. Nat Rev Genet 10:32-42. CrossRef Medline

Heisenberg M (2003) Mushroom body memoir: from maps to models. Nat Rev Neurosci 4:266-275. CrossRef Medline

Honjo K, Furukubo-Tokunaga K (2005) Induction of cAMP response element-binding protein-dependent medium-term memory by appetitive gustatory reinforcement in Drosophila larvae. J Neurosci 25:79057913. CrossRef Medline

Iijima K, Iijima-Ando K (2008) Drosophila models of Alzheimer's amyloidosis: the challenge of dissecting the complex mechanisms of toxicity of amyloid-beta 42. J Alzheimers Dis 15:523-540. CrossRef Medline

Jacobsen JS, Wu CC, Redwine JM, Comery TA, Arias R, Bowlby M, Martone R, Morrison JH, Pangalos MN, Reinhart PH, Bloom FE (2006) Earlyonset behavioral and synaptic deficits in a mouse model of Alzheimer's disease. Proc Natl Acad Sci U S A 103:5161-5166. CrossRef Medline

Jan LY, Papazian DM, Timpe L, O'Farrell P, Jan YN (1985) Application of Drosophila in molecular genetics in the study of neural function; studies of the shaker locus for a potassium channel. Trends Neurosci 8:234-238. CrossRef

Johnson AA, Sarthi J, Pirooznia SK, Reube W, Elefant F (2013) Increasing Tip60 HAT levels rescues axonal transport defects and associated behavioral phenotypes in a Drosophila Alzheimer's disease model. J Neurosci 33:7535-7547. CrossRef Medline

Kim TK, Hemberg M, Gray JM, Costa AM, Bear DM, Wu J, Harmin DA, Laptewicz M, Barbara-Haley K, Kuersten S, Markenscoff-Papadimitriou E, Kuhl D, Bito H, Worley PF, Kreiman G, Greenberg ME (2010) Widespread transcription at neuronal activity-regulated enhancers. Nature 465:182-187. CrossRef Medline

Langley B, Gensert JM, Beal MF, Ratan RR (2005) Remodeling chromatin and stress resistance in the central nervous system: histone deacetylase inhibitors as novel and broadly effective neuroprotective agents. Curr Drug Targets CNS Neurol Disord 4:41-50. CrossRef Medline

Leidinger P, Backes C, Deutscher S, Schmitt K, Mueller SC, Frese K, Haas J, Ruprecht K, Paul F, Stähler C, Lang CJ, Meder B, Bartfai T, Meese E, Keller A (2013) A blood based 12-miRNA signature of Alzheimer disease patients. Genome Biol 14:R78. CrossRef Medline

Levenson JM, O'Riordan KJ, Brown KD, Trinh MA, Molfese DL, Sweatt JD (2004) Regulation of histone acetylation during memory formation in the hippocampus. J Biol Chem 279:40545-40559. CrossRef Medline

Lorbeck M, Pirooznia K, Sarthi J, Zhu X, Elefant F (2011) Microarray analysis uncovers a role for Tip60 in nervous system function and general metabolism. PLoS One 6:e18412. CrossRef Medline

Lu T, Aron L, Zullo J, Pan Y, Kim H, Chen Y, Yang TH, Kim HM, Drake D, Liu XS, Bennett DA, Colaiácovo MP, Yankner BA (2014) REST and stress resistance in ageing and Alzheimer's disease. Nature 507:448-454. CrossRef Medline

Lu X, Wang L, Yu C, Yu D, Yu G (2015) Histone acetylation modifiers in the pathogenesis of Alzheimer's disease. Front Cell Neurosci 9:226. CrossRef Medline

Meaney MJ, Ferguson-Smith AC (2010) Epigenetic regulation of the neural transcriptome: the meaning of the marks. Nat Neurosci 13:1313-1318. CrossRef Medline

Miech C, Pauer HU, He X, Schwarz TL (2008) Presynaptic local signaling by a canonical wingless pathway regulates development of the Drosophila neuromuscular junction. J Neurosci 28:10875-10884. CrossRef Medline

Minhas S, Khanum A, Riaz F, Khan S, Alvi A (2017) Predicting progression from mild cognitive impairment to Alzheimer's disease using autoregressive modelling of longitudinal and multimodal biomarkers. IEEE J Biomed Health Inform. In press. Available at https://ieeexplore.ieee.org/ document/7929276/.

Narayan PJ, Lill C, Faull R, Curtis MA, Dragunow M (2015) Increased acetyl and total histone levels in post-mortem Alzheimer's disease brain. Neurobiol Dis 74:281-294. CrossRef Medline

Nelson ED, Monteggia LM (2011) Epigenetics in the mature mammalian brain: effects on behavior and synaptic transmission. Neurobiol Learn Mem 96:53-60. CrossRef Medline

Packard M, Koo ES, Gorczyca M, Sharpe J, Cumberledge S, Budnik V (2002) The Drosophila wnt, wingless, provides an essential signal for pre- and postsynaptic differentiation. Cell 111:319-330. CrossRef Medline

Peixoto L, Abel T (2013) The role of histone acetylation in memory formation and cognitive impairments. Neuropsychopharmacology 38:62-76. CrossRef Medline

Peleg S, Sananbenesi F, Zovoilis A, Burkhardt S, Bahari-Javan S, Agis-Balboa RC, Cota P, Wittnam JL, Gogol-Doering A, Opitz L, Salinas-Riester G, Dettenhofer M, Kang H, Farinelli L, Chen W, Fischer A (2010) Altered histone acetylation is associated with age-dependent memory impairment in mice. Science 328:753-756. CrossRef Medline

Peng IF, Berke BA, Zhu Y, Lee WH, Chen W, Wu CF (2007) Temperaturedependent developmental plasticity of Drosophila neurons: cellautonomous roles of membrane excitability, $\mathrm{Ca} 2+$ influx, and cAMP signaling. J Neurosci 27:12611-12622. CrossRef Medline

Petersen RC, Roberts RO, Knopman DS, Boeve BF, Geda YE, Ivnik RJ, Smith GE, Jack CR Jr (2009) Mild cognitive impairment: ten years later. Arch Neurol 66:1447-1455. CrossRef Medline

Pirooznia SK, Chiu K, Chan MT, Zimmerman JE, Elefant F (2012a) Epigenetic regulation of axonal growth of Drosophila pacemaker cells by histone acetyltransferase tip60 controls sleep. Genetics 192:1327-1345. CrossRef Medline

Pirooznia SK, Sarthi J, Johnson AA, Toth MS, Chiu K, Koduri S, Elefant F (2012b) Tip60 HAT activity mediates APP induced lethality and apoptotic cell death in the CNS of a Drosophila Alzheimer's disease model. PLoS One 7:e41776. CrossRef Medline

Riccio A (2010) Dynamic epigenetic regulation in neurons: enzymes, stimuli and signaling pathways. Nat Neurosci 13:1330-1337. CrossRef Medline

Roos J, Hummel T, Ng N, Klämbt C, Davis GW (2000) Drosophila futsch regulates synaptic microtubule organization and is necessary for synaptic growth. Neuron 26:371-382. CrossRef Medline

Roth TL, Roth ED, Sweatt JD (2010) Epigenetic regulation of genes in learning and memory. Essays Biochem 48:263-274. CrossRef Medline

Rouaux C, Jokic N, Mbebi C, Boutillier S, Loeffler JP, Boutillier AL (2003) 
Critical loss of $\mathrm{CBP} / \mathrm{p} 300$ histone acetylase activity by caspase- 6 during neurodegeneration. EMBO J 22:6537-6549. CrossRef Medline

Sarthi J, Elefant F (2011) dTip60 HAT activity controls synaptic bouton expansion at the Drosophila neuromuscular junction. PLoS One 6:e26202. CrossRef Medline

Selvi BR, Cassel JC, Kundu TK, Boutillier AL (2010) Tuning acetylation levels with HAT activators: therapeutic strategy in neurodegenerative diseases. Biochim Biophys Acta 1799:840 - 853. CrossRef Medline

Steele JW, Brautigam H, Short JA, Sowa A, Shi M, Yadav A, Weaver CM, Westaway D, Fraser PE, St George-Hyslop PH, Gandy S, Hof PR, Dickstein DL (2014) Early fear memory defects are associated with altered synaptic plasticity and molecular architecture in the TgCRND8 Alzheimer's disease mouse model. J Comp Neurol 522:2319-2335. CrossRef Medline

Stokin GB, Lillo C, Falzone TL, Brusch RG, Rockenstein E, Mount SL, Raman R, Davies P, Masliah E, Williams DS, Goldstein LS (2005) Axonopathy and transport deficits early in the pathogenesis of Alzheimer's disease. Science 307:1282-1288. CrossRef Medline

Tea JS, Chihara T, Luo L (2010) Histone deacetylase Rpd3 regulates olfac- tory projection neuron dendrite targeting via the transcription factor prospero. J Neurosci 30:9939-9946. CrossRef Medline

Torroja L, Packard M, Gorczyca M, White K, Budnik V (1999) The Drosophila beta-amyloid precursor protein homolog promotes synapse differentiation at the neuromuscular junction. J Neurosci 19:7793-7803. CrossRef Medline

Tully T, Preat T, Boynton SC, Del Vecchio M (1994) Genetic dissection of consolidated memory in Drosophila. Cell 79:35-47. CrossRef Medline

Xu S, Wilf R, Menon T, Panikker P, Sarthi J, Elefant F (2014) Epigenetic control of learning and memory in Drosophila by Tip60 HAT action. Genetics 198:1571-1586. CrossRef Medline

Zhang B, Gaiteri C, Bodea LG, Wang Z, McElwee J, Podtelezhnikov AA, Zhang C, Xie T, Tran L, Dobrin R, Fluder E, Clurman B, Melquist S, Narayanan M, Suver C, Shah H, Mahajan M, Gillis T, Mysore J, MacDonald ME, et al. (2013) Integrated systems approach identifies genetic nodes and networks in late-onset Alzheimer's disease. Cell 153:707-720. CrossRef Medline

Zhang K, Schrag M, Crofton A, Trivedi R, Vinters H, Kirsch W (2012) Targeted proteomics for quantification of histone acetylation in Alzheimer's disease. Proteomics 12:1261-1268. CrossRef Medline 